\title{
Synthesis and antitumor activity of aromatic camptothecin esters
}

\author{
ZHISONG CAO, JOHN MENDOZA, ALBERT DEJESUS, DANA VARDEMAN and BEPPINO GIOVANELLA \\ The Christus Stehlin Foundation for Cancer Research, 1918 Chenevert Street, \\ Houston, TX 77003, USA
}

Received October 31, 2007; Accepted December 27, 2007

\begin{abstract}
Twenty-eight new aromatic esters of camptothecins 2-29 were prepared in yields of 5 to $96 \%$ by straight acylation of camptothecin (1a) and 9-nitrocamptothecin (1b) with various aromatic acids as acylating agents. All of these esters were tested against 14 different human cancer cell lines. The antitumor activity of these compounds was related to the nature of the substituting groups of their side aromatic chains. In general, esters with strong electron-withdrawing groups on their side aromatic chains were active; esters with halogen-substituted side aromatic chains were slightly active; and esters without any substituting groups on their side aromatic chains were practically inactive. The $\mathrm{IC}_{50}$ studies showed that the majority of these esters were not as potent as their parental compounds $\mathbf{1 a}$ and $\mathbf{1 b}$; whereas, the potencies of esters $\mathbf{6}$ and $\mathbf{2 5}$ were exceptionally high, much higher than the commercial camptothecin analogues and comparable to (or slightly more potent than) their parental compounds.
\end{abstract}

\section{Introduction}

Camptothecin, a natural product originally found in China, was isolated and purified by Wall and his co-workers in 1966 (1). Since it demonstrated effective anticancer activity in animal models, camptothecin was quickly tested in human clinical trials. Unfortunately, no anticancer activity was found; instead, severe toxicities were observed in patients (2-6). Trials were accordingly discontinued. The reason for the failure of the early trials was later found to be the incorrect drug formulation selected. As it is insoluble in water, camptothecin was converted to its sodium salt form for intravenous administration. This form of the molecule, although water-soluble, is practically devoid of anticancer activity. A careful evaluation of these agents in animal

Correspondence to: Dr Zhisong Cao, The Stehlin Foundation for Cancer Research, 1918 Chenevert Street, Houston, TX 77003, USA E-mail: zcao@stehlin.org

Key words: alkaloids, camptothecin, chemical synthesis, structureactivity relationship models made by Wani et al revealed that the sodium salt has only one-tenth the potency of the parent compound (7). Important parameters for the anticancer activity of camptothecin derivatives have now been established (8), showing that the intact lactone form with an $\alpha$-hydroxyl group at position 20 of the molecule with an $S$-configuration is required for antitumor activity.

In our laboratory, camptothecin and its semi-synthetic derivative 9-nitrocamptothecin, have shown significant activity against a wide spectrum of human tumors grown as xenografts in nude mice $(9,10)$, but considerably less activity for these two compounds was observed in human clinical trials. This difference in antitumor activity between humans and mice has been associated with the finding that the opening of the lactone of the molecule to its carboxylate form is much more advanced in humans. For example, $~ 50 \%$ of 9-nitrocamptothecin is present as the lactone form in mice, but only $2-5 \%$ of the molecule can be found as the lactone form in humans (unpublished data). Further studies on the stability of camptothecin derivatives in human serum have been conducted by Burke (11).

Clearly, there is a need for a camptothecin derivative which is able to maintain the molecule as an intact lactone when circulating in the human body. A number of attempts at improving the lactone stability of camptothecins have been undertaken, of which acylation of 20-OH of the molecule has proven to be the most efficient. For example, Zhao and his co-workers reported that 20 -acyl derivatives of camptothecin were substantially more stable in lactone form than their $20-\mathrm{OH}$ parents and found that 20-O-acyl camptothecin derivatives remained unaffected even at $\mathrm{pH} 9.5$ (12), whereas the lactone of parental camptothecin molecules underwent hydrolysis reaction to form carboxylate salt at physiological $\mathrm{pH} 7.4(13,14)$. We previously reported the preparation of camptothecin esters by acylating the $20-\mathrm{OH}$ group with organic anhydrides, which resulted in significant increases in the stability of ester compounds in the human body, and the presence of much higher concentrations of the lactone form of the molecules (15).

The SAR studies with our synthetic 20-acyl camptothecin derivatives showed that all alkyl esters had low toxicity, and that their antitumor activities varied depending on the property of their side ester chains $(16,17)$. Only compounds with a straight $\mathrm{C}_{2}$ or $\mathrm{C}_{3}$ alkyl ester chain were found to be active; the remainder were either slightly active or not active 
at all. Alkyl camptothecin esters are not independently active. They are activated by a group of enzymes called esterases. Camptosar (CPT-11), a clinically useful anticancer agent, is a prodrug of 10-hydroxy-7-ethylcamptothecin (SN38). A number of studies have shown that, in various mammals and humans, camptosar is converted to its active metabolite SN-38 by liver carboxylesterase (18-21). Carboxylesterase enzymes exist in different organs and tissues. Many of these enzymes have been purified and characterized. For example, Mentlein et al and Meintlein and Heymann purified five carboxylesterases from rat liver microsomes, investigated their activity, and found that these enzymes had high carboxylesterase activity with simple aliphatic and aromatic esters $(22,23)$. Our results are consistent with Mentlein's observations, that is, only those simple alkyl camptothecin esters with a $\mathrm{C}_{2}$ or $\mathrm{C}_{3}$ chain have significant antitumor activity. In order to obtain optimal camptothecin ester derivatives with high effectiveness and low toxicity for clinical use, we prepared many aryl camptothecin esters. We also assayed some of their biological properties and obtained some notable results, which we report in this paper.

\section{Materials and methods}

Dry nitrogen was routinely used as the reaction atmosphere in all reactions. All glassware was baked at $70 \pm 10^{\circ} \mathrm{C}$ for a minimum of $2 \mathrm{~h}$ before being used. Melting points were obtained with a Mel-Temp melting point apparatus and were uncorrected. The ${ }^{1} \mathrm{H}$ NMR spectrum of approximately $10 \%$ (w/v) solution in $\mathrm{CDCl}_{3}$ or DMSO was obtained at $270.05 \mathrm{MHz}$ with a Jeol GX-270 WB NMR spectrometer. Chemical shifts are reported in parts per million ( $\delta$ scale), employing tetramethylsilane as an internal standard. In reporting the NMR data, we used the following abbreviations: coupling constants in Hertz (J), singlet (s), doublet (d), triplet (t), broad singlet (bs) and multiplet $(\mathrm{m})$. Mass spectra were recorded using a VG ZAB-SEQ mass spectrometer (VG Analytical Co., UK) with a resolution of 10,000 . Routinely used solvents such as THF and methylene chloride were dried and freshly distilled. Silica gel (230-400 mesh, Aldrich) for column chromatography was used for all product separations. Eastman chromagram (silica gel with fluorescent indicator on polyethylene) sheets were employed in thinlayer chromatography (TLC) operations. The numbering system used in reporting NMR data is shown in Table I.

The dicyclohexylcarbodiimide(DCC)/dimethylaminopyridine (DMAP) reagent system is frequently used for the esterification reaction of carboxylic acids with alcohols. This procedure offers a convenient method for the acylation of alcohols and thiols with carboxylic acids under mild conditions (24-26). The success of the reaction depends on the high efficiency of dimethylaminopyridine as a nucleophilic catalyst in group transfer reactions (27). The acylation proceeds without the need of a pre-formed, activated carboxylic acid derivative, at room temperature, under nonacidic or mildly basic, conditions. A variety of aprotic solvents of comparable polarity such as methylene chloride, diethyl ether, tetrahydrofuran, and acetonitrile can be used. Two research groups used this method to successfully prepare water-soluble camptothecin glycinates $(28,29)$. We also used this chemistry to synthesize our aromatic esters of camptothecins.

Camptothecin (1a) and 9-nitrocamptothecin (1b) were allowed to react with aromatic carboxylic acids and dicyclohexylcarbodiimide with DMAP as the catalyst to give the corresponding ester products $\mathbf{2 - 2 9}$ as depicted in Fig. 1. The reaction yields varied from 5 to $96 \%$ depending on the nature of the substituting group on the benzene ring of carboxylic acids. For example, the reaction of $\mathbf{1 b}$ with 2-hydroxyl benzoic acid gave product $\mathbf{2 3}$ in only a $5 \%$ yield; while the reaction of 1a with p-nitrobenzoic acid gave product $\mathbf{7}$ in a $96 \%$ yield. The starting aromatic acids react with dicyclohexyldiimide under room temperature with dimetylaminopyridine as the catalyst to form intermediate product $\mathbf{A}$. The subsequent nucleophilic substitution of $\mathbf{A}$ with $\mathbf{1 a}$ or $\mathbf{1 b}$ gives ester products. When the Ar group has an electron-donating group on its benzene ring, the carbonyl carbon of $\mathbf{A}$ becomes less cationic, which makes the nucleophilic substitution by 1a or 1b more difficult. When the Ar group of $\mathbf{A}$ has an electron-withdrawing group on its benzene ring, the nucleophilic substitution by $\mathbf{1 a}$ or $\mathbf{1 b}$ becomes easier due to the highly cationic nature of the carbonyl carbon of $\mathbf{A}$. A strong electron-donating group such as the $\mathrm{N}, \mathrm{N}$-dimethyl amino group can even prevent the reaction from occurring. For example, the reaction between camptothecin 1a and 4-N,N-dimethylaminobenzoic acid under the same reaction conditions did not produce any ester product; 1a was completely recovered. We only isolated one product for most reactions, but the reaction of 2-nitrobenzoic acid with 9-nitrocamptothecin $\mathbf{1 b}$ gave two isomeric esters, $\mathbf{1 0}$ and 29, in the same amount of yield (50/50) with a total yield of 9\%. As depicted in Fig. 2, attacking both sides of the carbonyl group of intermediate $\mathbf{A}$ by $\mathbf{1 b}$, produces isomeric esters $\mathbf{1 0}$ and 29 equally. Esters $\mathbf{1 0}$ and 29 are not convertible into each other as rotation around the bond $\mathrm{C}_{20}-\mathrm{O}-\mathrm{C}_{22}-\mathrm{C}_{23}$ is not possible due to the stereo hindrance between the C19-methyl group and the nitro group attaching on either $\mathrm{C}_{24}$ or $\mathrm{C}_{28}$.

The starting camptothecin was purchased from P.R. China and was purified before being used. The starting 9-nitrocamptothecin was prepared following an established procedure (30). All other chemicals were from Aldrich Chemical Co. (Milwaukee, WI) and used as purchased. Preparation of the new aromatic esters of the camptothecins, and the corresponding structural data of these new compounds are described compound by compound as follows.

Camptothecin-20-O-benzoate [2]. Camptothecin (0.8 g, $0.0023 \mathrm{~mol})$, benzoic acid $(1.8 \mathrm{~g}, 0.014 \mathrm{~mol})$, DCC (1.2 g, $0.0058 \mathrm{~mol})$, and DMAP $(0.3 \mathrm{~g}, 0.0025 \mathrm{~mol})$ were added to $60 \mathrm{ml} \mathrm{DMF}$ in a $250-\mathrm{ml}$ round-bottomed flask equipped with a mechanical stirrer. The mixture was stirred under $\mathrm{N}_{2}$ at room temperature for $72 \mathrm{~h}$. Dicyclohexyl urea formed during the reaction was removed by filtration. The filtrate was poured onto $600 \mathrm{ml}$ ice water while stirring. The stirring was maintained for $30 \mathrm{~min}$. After filtration the crude product was chromatographically separated with THF- $\mathrm{CH}_{2} \mathrm{Cl}_{2}(1: 15)$ as eluent. Pure product $2(0.4 \mathrm{~g})$ was obtained as a white powder after precipitation from petroleum ether, yield $38 \%$, purity 99\% (HPLC). ${ }^{1} \mathrm{H}$ NMR in DMSO: $\delta 1.13(3 \mathrm{H}, \mathrm{t}$, 


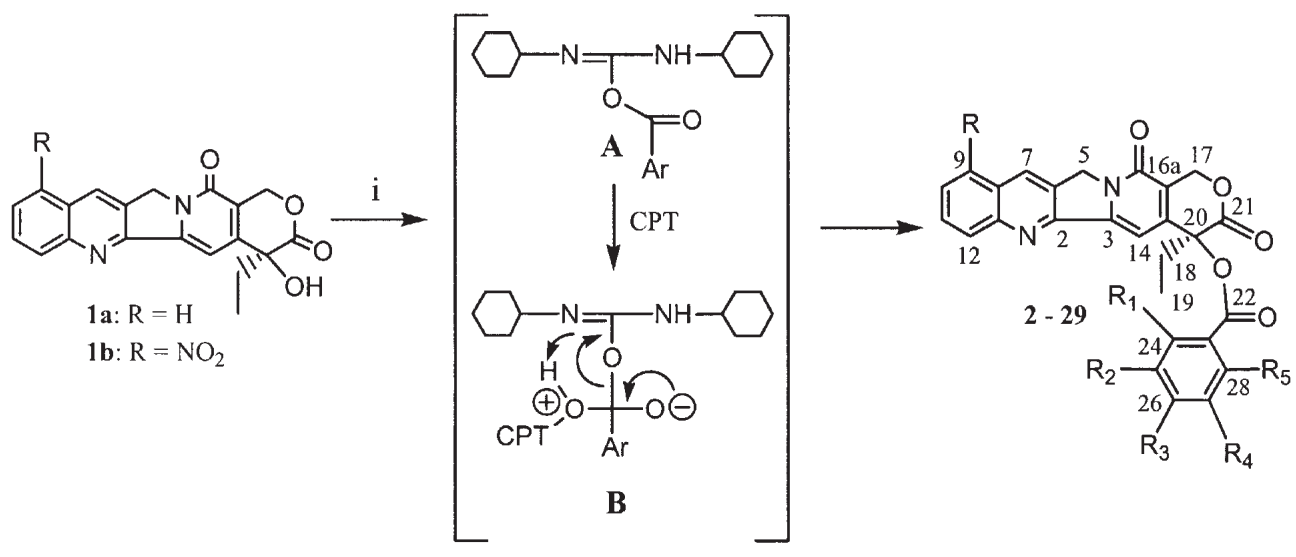

i. Aromatic carboxylic acid, DCC, DMAP, DMF, and rt

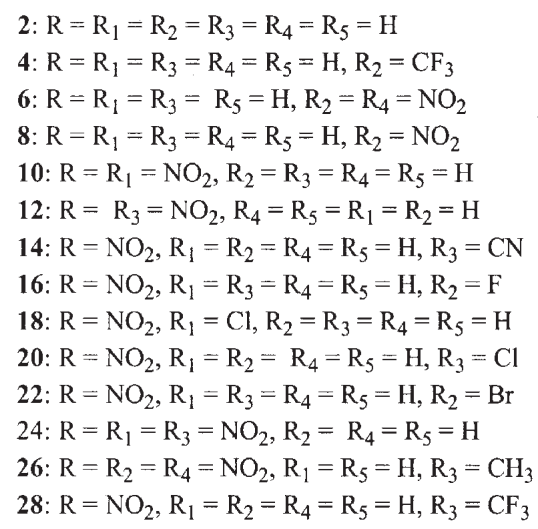

Figure 1. Ester products 2-29 derived from camptothecin and 9-nitrocamptothecin.

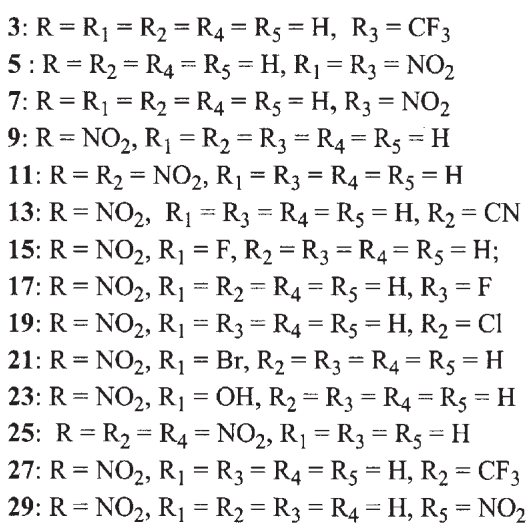

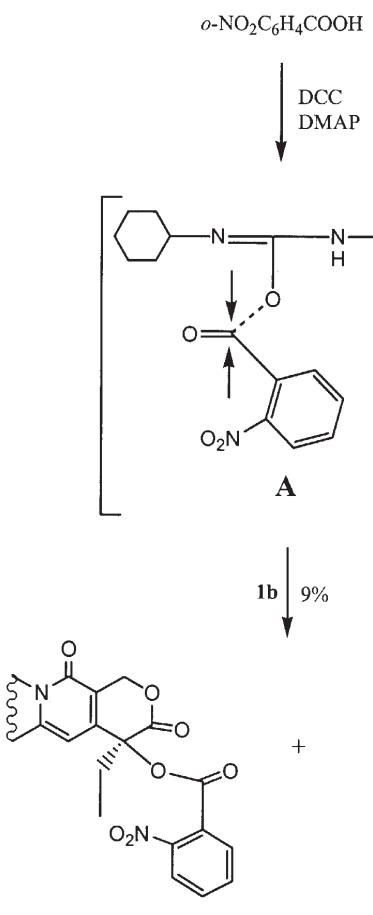

10

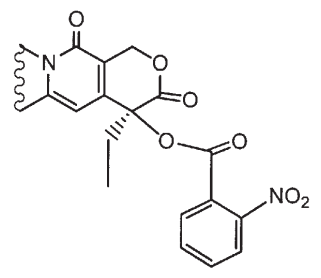

29
Figure 2. Isomeric esters $\mathbf{1 0}$ and $\mathbf{2 9}$ are produced in equal amounts by the reaction of 2-nitrobenzoic acid and 9-nitrocamptothecin.
$\mathrm{J}=7.04 \mathrm{~Hz}, \mathrm{C} 19$-methyl protons), 2.20-2.40 (2H, m, C18methylene protons), 5.28 ( $2 \mathrm{H}, \mathrm{s}, \mathrm{C} 5$-methylene protons), 5.56 $(2 \mathrm{H}, \mathrm{s}, \mathrm{C} 17-$ methylene protons), $7.05(1 \mathrm{H}, \mathrm{s}, \mathrm{C} 14-\mathrm{H}), 7.50$ 8.20 (9H, m, C9-C12-Hs and C23-C28-Hs), 8.68 (1H, s, C7$\mathrm{H}) ;{ }^{13} \mathrm{C}$ NMR (DMSO): $\delta 8.0$ (C19), 32.2 (C18), 49.0 (C5), 64.9 (C17), 74.8 (C20), 92.6 (C14), 117.5, 125.8, 126.0, 126.3, 126.8, 127.0, 127.2, 127.6, 128.0, 128.6, 130.1, 133.0, $144.1,144.8,146.3,150.5,155.0$ (C2, C3, C6-C13, C15, C16, C16a, C23-C28), 162.8, 165.9 (C21, C22); mass m/e (relative intensity): $452\left(\mathrm{~m}^{+}, 25\right), 330$ (100), 315 (35), 302 (60), 287 (40), 169 (12), 122 (36), 105 (70), 77 (35), 69 (26); precise mass $\left(\mathrm{C}_{27} \mathrm{H}_{20} \mathrm{~N}_{2} \mathrm{O}_{5}\right)$ : found, 452.137 ; required, 452.137

Camptothecin-20-O-p-triflouromethylbenzoate [3]. Using camptothecin $(0.8 \mathrm{~g}, 0.0023 \mathrm{~mol})$, trifluoro-p-toluic acid (1.6 g, $0.0084 \mathrm{~mol})$, DCC (1.2 g, $0.0058 \mathrm{~mol})$, DMAP (0.3 g, $0.0025 \mathrm{~mol})$ as the starting materials, pure product $3(1 \mathrm{~g})$ was obtained as a white powder, yield $84 \%$, purity $99 \%$ (HPLC). ${ }^{1} \mathrm{H}$ NMR (DMSO): $\delta 1.11$ (3H, t, J=7.10 Hz, C19methyl protons), 2.25-2.50 (2H, m, C18-methylene protons), 5.28 ( $2 \mathrm{H}, \mathrm{s}, \mathrm{C} 5$-methylene protons), 5.56 (2H, s, C17-methylene protons), $7.12(1 \mathrm{H}, \mathrm{s}, \mathrm{C} 14-\mathrm{H}), 7.66(1 \mathrm{H}, \mathrm{t}, \mathrm{J}=8.50 \mathrm{~Hz}$, C10-H), $7.80(1 \mathrm{H}, \mathrm{t}, \mathrm{J}=8.48 \mathrm{~Hz}, \mathrm{C} 11-\mathrm{H}), 8.00(2 \mathrm{H}, \mathrm{d}, \mathrm{J}=8.46 \mathrm{~Hz}$, C9-H, C12-H), 8.12 (2H, d, J=8.38 Hz, C24-H, C28-H), 8.35 
$(2 \mathrm{H}, \mathrm{d}, \mathrm{J}=8.34 \mathrm{~Hz}, \mathrm{C} 25-\mathrm{H}, \mathrm{C} 27-\mathrm{H}), 8.68(1 \mathrm{H}, \mathrm{s}, \mathrm{C} 7-\mathrm{H}) ;{ }^{13} \mathrm{C}$ NMR (DMSO): $\delta 7.6$ (C19), 30.5 (C18), $47.5\left(\mathrm{C} 26-\underline{-F}_{3}\right)$, 50.4 (C5), 66.5 (C17), 77.1 (C20), 119.0, 126.0, 127.6, $127.8,128.2,128.8,129.7,130.1,130.6,131.4,132.0,145.0$, 146.3, 147.3, 152.0, 156.5 (C2, C3, C6-C13, C15, C16, C16a, C23-C28), 163.0, 166.5 (C21, C22); mass m/e (relative intensity): $520\left(\mathrm{~m}^{+}, 45\right), 330$ (100), $315(20), 302$ (78), 173 (40), 147 (9), 56 (12); precise mass $\left(\mathrm{C}_{28} \mathrm{H}_{19} \mathrm{~N}_{2} \mathrm{O}_{5} \mathrm{~F}_{3}\right)$ : found, 520.124; required, 520.125.

Camptothecin-20-O-m-triflouromethylbenzoate [4]. Using camptothecin $(0.8 \mathrm{~g}, 0.0023 \mathrm{~mol})$, trifluoro-m-toluic acid $(1.7 \mathrm{~g}, 0.0089 \mathrm{~mol})$, DCC $(1.2 \mathrm{~g}, 0.0058 \mathrm{~mol})$, and DMAP $(0.3 \mathrm{~g}, 0.0025 \mathrm{~mol})$ as the starting materials, pure product 4 $(1.07 \mathrm{~g})$ was obtained as a white powder, yield $89 \%$, purity 99\% (HPLC). ${ }^{1} \mathrm{H}$ NMR (DMSO): $\delta 1.08$ (3H, t, J=7.08 Hz, C19-methyl protons), 2.30-2.50 (2H, m, C18-methylene protons), $5.28(2 \mathrm{H}, \mathrm{s}, \mathrm{C}$-methylene protons), $5.58(2 \mathrm{H}, \mathrm{s}$, C17-methylene protons), $7.18(1 \mathrm{H}, \mathrm{s}, \mathrm{C} 14-\mathrm{H}), 7.66(1 \mathrm{H}, \mathrm{t}$, $\mathrm{J}=8.14 \mathrm{~Hz}, \mathrm{C} 10-\mathrm{H}), 7.80(1 \mathrm{H}, \mathrm{t}, \mathrm{J}=8.09 \mathrm{~Hz}, \mathrm{C} 11-\mathrm{H}), 7.90$ $(1 \mathrm{H}, \mathrm{t}, \mathrm{J}=8.08 \mathrm{~Hz}, \mathrm{C} 25-\mathrm{H}), 8.08[2 \mathrm{H}, \mathrm{t}(\mathrm{d}+\mathrm{d}), \mathrm{J}=8.08 \mathrm{~Hz}, \mathrm{C} 9-\mathrm{H}$, C12-H], $8.16(1 \mathrm{H}, \mathrm{d}, \mathrm{J}=8.07 \mathrm{~Hz}, \mathrm{C} 24-\mathrm{H}), 8.36(1 \mathrm{H}, \mathrm{s}, \mathrm{C} 28-\mathrm{H})$, $8.44(1 \mathrm{H}, \mathrm{d}, \mathrm{J}=8.08 \mathrm{~Hz}, \mathrm{C} 26-\mathrm{H}), 8.68(1 \mathrm{H}, \mathrm{s}, \mathrm{C} 7-\mathrm{H}) ;{ }^{13} \mathrm{C}$ NMR (DMSO): $\delta 7.6$ (C19), 33.0 (C18), 46.5 (C27-CF3), 47.2 (C5), 65.0 (C17), 76.0 (C20), 95.5 (C14), 117.0, 124.5, 126.0, 126.2, 127.0, 127.1, 127.5, 128.0, 128.5, 128.8, 129.0, $129.5,132.2,143.2,144.5,146.0,150.8,155.2$ (C2, C3, C6C13, C15, C16, C16a, C23-C28), 161.5, 165.5 (C21, C22); mass $\mathrm{m} / \mathrm{e}$ (relative intensity): $520\left(\mathrm{~m}^{+}, 50\right), 330(100), 315$ (40), 302 (97), 287 (38), 246 (6), 190 (10), 173 (56), 145 (38), 124 (3), 75 (3); precise mass $\left(\mathrm{C}_{28} \mathrm{H}_{19} \mathrm{~N}_{5} \mathrm{~F}_{3}\right)$ : found, 520.125; required, 520.125 .

Camptothecin-20-O-o,p-dinitrobenzoate [5]. Using camptothecin $(0.8 \mathrm{~g}, 0.0023 \mathrm{~mol}), 2,4-$ dinitrobenzoic acid $(2 \mathrm{~g}$, $0.0094 \mathrm{~mol})$, DCC (1.3 g, $0.0063 \mathrm{~mol})$, and DMAP (0.3 g, $0.0025 \mathrm{~mol})$ as the starting materials, pure product $5(0.13 \mathrm{~g})$ was obtained as a white powder, yield $10 \%$, purity $99 \%$ (HPLC). ${ }^{1} \mathrm{H}$ NMR in $\mathrm{CDCl}_{3}: \delta 1.10(3 \mathrm{H}, \mathrm{t}, \mathrm{J}=7.06 \mathrm{~Hz}, \mathrm{C} 19-$ methyl protons), 2.20-2.45 ( $2 \mathrm{H}, \mathrm{m}, \mathrm{C} 18$-methylene protons), $5.32(2 \mathrm{H}, \mathrm{s}, \mathrm{C} 5$-methylene protons $), 5.40-5.85(2 \mathrm{H}, \mathrm{dd}$, $\mathrm{J}=18.50,18.52 \mathrm{~Hz}, \mathrm{C} 17$-methylene protons), $7.53(1 \mathrm{H}, \mathrm{s}$, C14-H), $7.66(1 \mathrm{H}, \mathrm{t}, \mathrm{J}=8.05 \mathrm{~Hz}, \mathrm{C} 10-\mathrm{H}), 7.83(1 \mathrm{H}, \mathrm{t}, \mathrm{J}=8.07 \mathrm{~Hz}$, C11-H), $7.96(1 \mathrm{H}, \mathrm{d}, \mathrm{J}=8.08 \mathrm{~Hz}, \mathrm{C} 9-\mathrm{H}), 8.18(1 \mathrm{H}, \mathrm{d}, \mathrm{J}=8.07 \mathrm{~Hz}$, C12-H), $8.24(1 \mathrm{H}, \mathrm{d}, \mathrm{J}=8.05 \mathrm{~Hz}, \mathrm{C} 28-\mathrm{H}), 8.42(1 \mathrm{H}, \mathrm{s}, \mathrm{C} 25-\mathrm{H})$, $8.60(1 \mathrm{H}, \mathrm{d}, \mathrm{J}=8.06 \mathrm{~Hz}, \mathrm{C} 27-\mathrm{H}), 8.85(1 \mathrm{H}, \mathrm{s}, \mathrm{C} 7-\mathrm{H}) ;{ }^{13} \mathrm{C} \mathrm{NMR}$ $\left(\mathrm{CDCl}_{3}\right)$ : 87.8 (C19), 32.1 (C18), 50.3 (C5), 67.4 (C17), 78.4 (C20), 95.4 (C14), 120.2, 123,4, 128.3, 128.5, 129.4, 129.6, $130.6,131.3,132,4,144.5,146.6,148.6,152.1,157.3$ (C2, C3, C6-C13, C15, C16, C16a, C23-C28), 163.5, 166.7 (C21, $\mathrm{C} 22)$; mass $\mathrm{m} / \mathrm{e}$ (relative intensity): $542\left(\mathrm{~m}^{+}, 10\right), 330$ (100), 315 (38), 302 (78), 287 (42), 272 (12), 195 (10), 168 (40), 120 (20), 75 (18); precise mass $\left(\mathrm{C}_{27} \mathrm{H}_{18} \mathrm{~N}_{4} \mathrm{O}_{9}\right)$ : found, 542.107; required, 542.107 .

Camptothecin-20-O-m,m-dinitrobenzoate [6]. Using camptothecin $(0.8 \mathrm{~g}, 0.0023 \mathrm{~mol}), 3$, 5-dinitrobenzoic acid $(2 \mathrm{~g}, 0.0094 \mathrm{~mol})$, DCC $(1.3 \mathrm{~g}, 0.0063 \mathrm{~mol})$, and DMAP $(0.3 \mathrm{~g}$, $0.0025 \mathrm{~mol})$ as the starting materials, pure product $6(1.2 \mathrm{~g})$ was obtained as a white powder, yield $96 \%$, purity $99 \%$ (HPLC). ${ }^{1} \mathrm{H}$ NMR $\left(\mathrm{CDCl}_{3}\right): \delta 1.13(3 \mathrm{H}, \mathrm{t}, \mathrm{J}=7.03 \mathrm{~Hz}, \mathrm{C} 19-$ methyl protons), 2.30-2.60 (2H, m, C18-methylene protons), $5.35(2 \mathrm{H}, \mathrm{s}, \mathrm{C} 5$-methylene protons $), 5.45-5.85(2 \mathrm{H}, \mathrm{dd}$, $\mathrm{J}=17.90,17.98 \mathrm{~Hz}, \mathrm{C} 17$-methylene protons $), 7.20(1 \mathrm{H}, \mathrm{s}$, C14-H), $7.66(1 \mathrm{H}, \mathrm{t}, \mathrm{J}=8.06 \mathrm{~Hz}, \mathrm{C} 10-\mathrm{H}), 7.78(1 \mathrm{H}, \mathrm{t}, \mathrm{J}=8.04 \mathrm{~Hz}$, C11-H), $7.94(1 \mathrm{H}, \mathrm{d}, \mathrm{J}=8.06 \mathrm{~Hz}, \mathrm{C} 9-\mathrm{H}), 8.13(1 \mathrm{H}, \mathrm{d}, \mathrm{J}=8.05 \mathrm{~Hz}$, C12-H), 8.40 (1H, s, C26-H), 9.18 (2H, s, C24-H, C28-H), $9.26(1 \mathrm{H}, \mathrm{s}, \mathrm{C} 7-\mathrm{H}) ;{ }^{13} \mathrm{C}$ NMR $\left(\mathrm{CDCl}_{3}\right): \delta 7.9(\mathrm{C} 19), 32.0$ (C18), 50.5 (C5), 67.2 (C17), 78.3 (C20), 95.4 (C14), 120.2, $123.1,128.1,128.3,129.5,129.8,130.9,131.4,132.6,144.6$, $146.8,148.5,152.0,157.2$ (C2, C3, C6-C13, C15, C16, C16a, C23-C28), 161.8, 166.5 (C21, C22); mass m/e (relative intensity): $542\left(\mathrm{~m}^{+}, 4\right), 330(44), 317(20), 235$ (10), 212 (100), 195 (15), 150 (35), 93 (25), 75 (23); precise mass $\left(\mathrm{C}_{27} \mathrm{H}_{18} \mathrm{~N}_{4} \mathrm{O}_{9}\right)$ : found, 542.109; required, 542.107.

Camptothecin-20-O-p-nitrobenzoate [7]. Using camptothecin $(0.8 \mathrm{~g}, 0.0023 \mathrm{~mol})$, p-nitrobenzoic acid $(2 \mathrm{~g}, 0.0120 \mathrm{~mol})$, DCC (1.2 g, $0.0063 \mathrm{~mol})$, and DMAP $(0.3 \mathrm{~g}, 0.0025 \mathrm{~mol})$ as the starting materials, pure product $7(1.1 \mathrm{~g})$ was obtained as a white powder, yield $96 \%$, purity $99 \%$ (HPLC). ${ }^{1} \mathrm{H}$ NMR $\left(\mathrm{CDCl}_{3}\right): \delta 1.12(3 \mathrm{H}, \mathrm{t}, \mathrm{J}=7.06 \mathrm{~Hz}, \mathrm{C} 19$-methyl protons), 2.30-2.60 (2H, m, C18-methylene protons), $5.50(2 \mathrm{H}, \mathrm{s}, \mathrm{C} 5-$ methylene protons), 5.71-6.10 ( $2 \mathrm{H}$, dd, J=18.01, $19.04 \mathrm{~Hz}$, C17-methylene protons), $7.23(1 \mathrm{H}, \mathrm{s}, \mathrm{C} 14-\mathrm{H}), 7.78(1 \mathrm{~h}, \mathrm{t}$, $\mathrm{J}=8.05 \mathrm{~Hz}, \mathrm{C} 10-\mathrm{H}), 7.98(1 \mathrm{H}, \mathrm{t}, \mathrm{J}=8.06 \mathrm{~Hz}, \mathrm{C} 11-\mathrm{H}), 8.05$ $(1 \mathrm{H}, \mathrm{d}, \mathrm{J}=8.04 \mathrm{~Hz}, \mathrm{C} 9-\mathrm{H}), 8.21(1 \mathrm{H}, \mathrm{d}, \mathrm{J}=8.06 \mathrm{~Hz}, \mathrm{C} 12-\mathrm{H})$, 8.30-8.60 (4H, m, C24-H, C25-H, C27-H, C28-H), $9.10(1 \mathrm{H}$, s, C7-H); ${ }^{13} \mathrm{C}$ NMR $\left(\mathrm{CDCl}_{3}\right)$ : $\delta 7.9$ (C19), 32.0 (C18), 50.2 (C5), 67.3 (C17), C20 buried in the area of solvent peaks, 95.8 (C14), 121.5, 123.8, 128.3, 128.5, 129.5, 131.0, 131.5, 134.0, 145.2, 146.5, 148.8, 151.1, 152.0, 156.2, 157.1 (C2, C3, C6-C13, C15, C16, C16a, C23-C28), 163.6, 167.0 (C21, $\mathrm{C} 22)$; mass $\mathrm{m} / \mathrm{e}$ (relative intensity): $497\left(\mathrm{~m}^{+}, 35\right), 330(100)$, 315 (30), 302 (86), 287 (38), 205 (8), 179 (12), 113 (16), 100 (35), 65 (20); precise mass $\left(\mathrm{C}_{27} \mathrm{H}_{19} \mathrm{~N}_{3} \mathrm{O}_{7}\right)$ : found, 497.122, required, 497.122 .

Camptothecin-20-O-m-nitrobenzoate [8]. Using camptothecin $(0.8 \mathrm{~g}, 0.0023 \mathrm{~mol})$, m-nitrobenzoic acid $(2 \mathrm{~g}, 0.0120 \mathrm{~mol})$, DCC $(1.2 \mathrm{~g}, 0.0058 \mathrm{~mol})$, and DMAP $(0.3 \mathrm{~g}, 0.0025 \mathrm{~mol})$ as the starting materials, pure product $8(1.1 \mathrm{~g})$ was obtained as a white powder, yield $96 \%$, purity $99 \%$ (HPLC). ${ }^{1} \mathrm{H}$ NMR $\left(\mathrm{CDCl}_{3}\right): \delta 1.12$ (3H, t, J=7.08Hz, C19-methyl protons), 2.20$2.60(2 \mathrm{H}, \mathrm{m}, \mathrm{C} 18$-methylene protons $), 5.30(2 \mathrm{H}, \mathrm{s}, \mathrm{C} 5-$ methylene protons), $5.40-5.82(2 \mathrm{H}, \mathrm{dd}, \mathrm{J}=17.53,17.56 \mathrm{~Hz}$, C17-methylene protons), 7.24 (1H, s, C14-H), 7.56-7.86 (3H, $\mathrm{m}, \mathrm{C} 10-\mathrm{H}, \mathrm{C} 11-\mathrm{H}, \mathrm{C} 25-\mathrm{H}), 7.88-8.20(2 \mathrm{H}, \mathrm{dd}, \mathrm{J}=8.05$, $8.07 \mathrm{~Hz}, \mathrm{C} 9-\mathrm{H}, \mathrm{C} 12-\mathrm{H}), 8.3-8.55$ (3H, m, C24-H, C26- $\mathrm{H}$, $\mathrm{C} 28-\mathrm{H}), 8.95(1 \mathrm{H}, \mathrm{s}, \mathrm{C} 7-\mathrm{H}) .{ }^{13} \mathrm{C}$ NMR $\left(\mathrm{CDCl}_{3}\right)$ : $\delta 7.9$ (C19), 31.8 (C18), 49.8 (C5), 66.7 (C17), C20 buried in solvent peaks, 95.5 (C14), 120.4, 1224.5, 124.6, 127.7, 127.9, 128.1, $129.5,129.7,129.8,130.1,130.5,130.9,135.7,145.0,146.4$, 148.1, 148.5, 151.9, 159.0 (C2, C3, C6-C13, C15, C16, C16a, C23-C28), 163.0, 166.9 (C21, C22). Mass m/e (relative intensity): $497\left(\mathrm{~m}^{+}, 6\right), 330(28), 315$ (12), 302 (18), 287 (15), 167 (100), 121 (40), 100 (10), 65 (35); precise mass $\left(\mathrm{C}_{27} \mathrm{H}_{19} \mathrm{~N}_{3} \mathrm{O}_{7}\right)$ : found, 497.122; required, 497.122. 
9-Nitrocamptothecin-20-O-benzoate [9]. Using 9-nitrocamptothecin $(1.5 \mathrm{~g}, 0.0038 \mathrm{~mol})$, benzoic acid $(1 \mathrm{~g}, 0.0082 \mathrm{~mol})$, DCC (1.7 g, $0.0083 \mathrm{~mol})$, and DMAP $(0.3 \mathrm{~g}, 0.0025 \mathrm{~mol})$ as the starting materials, pure product $9(0.2 \mathrm{~g})$ was obtained as a yellow powder, yield $11 \%$, purity $99 \%$ (HPLC). ${ }^{1} \mathrm{H}$ NMR $\left(\mathrm{CDCl}_{3}\right): \delta 1.10$ (3h, t, J=7.05 Hz, C19-methyl protons), 2.20$2.45(2 \mathrm{H}, \mathrm{m}, \mathrm{C} 18$-methylene protons $), 5.36(2 \mathrm{H}, \mathrm{s}, \mathrm{C} 5-$ methylene protons), 5.48-5.85 (2H, dd, J=18.03, $18.05 \mathrm{~Hz}$, C17-methylene protons), $7.21(1 \mathrm{H}, \mathrm{s}, \mathrm{C} 14-\mathrm{H}), 7.60-8.60(8 \mathrm{H}$, $\mathrm{m}, \mathrm{C} 10-\mathrm{C} 12-\mathrm{Hs}$, and C24-C28-Hs), $9.22(1 \mathrm{H}, \mathrm{s}, \mathrm{C} 7-\mathrm{H}) ;{ }^{13} \mathrm{C}$ NMR $\left(\mathrm{CDCl}_{3}\right)$ : $\delta 7.9(\mathrm{C} 19), 32.1$ (C18), $50.6(\mathrm{C} 5), 67.2$ (C17), 78.3 (C20), 97,6 (C14), 121.0, 124.1, 126.3, 127.2, 128.1, 131.1, 131.5, 132.5, 133.0, 137.0, 145.2, 145.6, 149.1, 154.2, 157.3 (C2, C3, C6-C13, C15, C16, C16a, C23-C28), 163.8, 166.9 (C21, C22); mass m/e (relative intensity): 497 $\left(\mathrm{m}^{+}, 10\right), 392$ (6), 375 (100), 360 (35), 347 (80), 332 (30), 319 (15), 302 (10), 286 (20), 274 (8), 258 (5), 216 (7); precise mass $\left(\mathrm{C}_{27} \mathrm{H}_{19} \mathrm{~N}_{3} \mathrm{O}_{7}\right)$ : found, 497.123; required, 497.122.

9-Nitrocamptothecin-20-O-o-nitrobenzoate [isomers 10 and 29]. Using 9-nitrocamptothecin (0.8 g, $0.0020 \mathrm{~mol}), 2$-nitrobenzoic acid $(0.8 \mathrm{~g}, 0.0048 \mathrm{~mol})$, DCC (1 g, $0.0049 \mathrm{~mol})$, and DMAP $(0.2 \mathrm{~g}, 0.0016 \mathrm{~mol})$ as the starting materials, two isomers were obtained as a yellow powder, total yield $9 \%$. Isomer 10: ${ }^{1} \mathrm{H} \mathrm{NMR}\left(\mathrm{CDCl}_{3}\right)$ : $\delta 1.05(3 \mathrm{H}, \mathrm{t}, \mathrm{J}=7.08 \mathrm{~Hz}, \mathrm{C} 19$ methyl protons), 2.25-2.42 (2H, m, C18-methylene protons), $5.44(2 \mathrm{H}, \mathrm{s}, \mathrm{C} 5$-methylene protons $), 5.45-5.82(2 \mathrm{H}$, dd, $\mathrm{J}=17.51,17.58 \mathrm{~Hz}, \mathrm{C} 17$-methylene protons), 7.24 (1H, s, C14-H), 7.60-8.58 (7H, m, C10-C12-Hs, and C24-C27-Hs), $9.26(1 \mathrm{H}, \mathrm{s}, \mathrm{C} 7-\mathrm{H}) ;{ }^{13} \mathrm{CNMR}\left(\mathrm{CDCl}_{3}\right)$ : $\delta 7.9(\mathrm{C} 19), 32.0$ (C18), 50.8 (C5), 67.5 (C17), 78.0 (C20), 97.9 (C14), 121.1, 124.1, 126.0, 127.4, 128.7, 131.0, 131.4, 132.7, 133.5, 137.1, $145.5,145.8,149.0,154.0,157.5$ (C2, C3, C6-C13, C15, C16, C16a, C23-C28), 164.0, 167.0 (C21, C22); mass m/e (relative intensity): $542\left(\mathrm{~m}^{+}, 2\right), 389(38), 375(85), 347$ (100), 361 (35), 332 (80), 306 (55), 286 (36), 272 (25), 260 (16), 230 (18), 203 (12); precise mass $\left(\mathrm{C}_{27} \mathrm{H}_{18} \mathrm{~N}_{4} \mathrm{O}_{9}\right)$ : found, 542.108; required, 542.107. Isomer 29: ${ }^{1} \mathrm{H} \mathrm{NMR}\left(\mathrm{CDCl}_{3}\right): \delta$ 1.14 (3H, t, J=7.06 Hz, C19-methyl protons), 2.28-2.46 (2H, m, C18-methylene protons), $5.40(2 \mathrm{H}, \mathrm{s}, \mathrm{C} 5$-methylene protons), 5.45-5.84 (2H, dd, J=17.50, $17.55 \mathrm{~Hz}, \mathrm{C} 17-$ methylene protons), $7.25(1 \mathrm{H}, \mathrm{s}, \mathrm{C} 14-\mathrm{H}), 7.60-8.58(7 \mathrm{H}, \mathrm{m}$, C10-C12-Hs, and C25-C28-Hs), 9.35 (1H, s, C7-4); ${ }^{13} \mathrm{C}$ NMR $\left(\mathrm{CDCl}_{3}\right)$ : $\delta 7.9$ (C19), 32.1 (C18), 50.8 (C5), 67.5 (C17), 78.0 (C20), 96.6 (C14), 121.4, 124.0, 126.0, 127.5, 128.8, 131.1, $131.4,132.7,133.3,137.1,145.4,145.8,148.9,149.2,157.6$ (C2, C3, C6-C13, C15, C16, C16a, C23-C28), 164.0, 167.0 (C21,C22); mass m/e (relative intensity): $542\left(\mathrm{~m}^{+}, 2\right), 389$ (38), 375 (85), 347 (100), 361 (35), 332 (80), 306 (55), 286 (36), 272 (25), 260 (16), 230 (18), 203 (12); precise mass $\left(\mathrm{C}_{27} \mathrm{H}_{18} \mathrm{~N}_{4} \mathrm{O}_{9}\right)$ : found, 542.108; required, 542.107.

9-Nitrocamptothecin-20-O-m-nitrobenzoate [11]. Using 9-nitrocamptothecin (0.6 g, $0.0015 \mathrm{~mol})$, 3-nitrobenzoic acid $(0.8 \mathrm{~g}, 0.0048 \mathrm{~mol})$, DCC (1 g, $0.0049 \mathrm{~mol})$, and DMAP $(0.2 \mathrm{~g}, 0.0016 \mathrm{~mol})$ as the starting materials, pure product $\mathbf{1 1}$ $(0.3 \mathrm{~g})$ was obtained as a yellow powder, yield $37 \%$, purity 99\% (HPLC). ${ }^{1} \mathrm{H}$ NMR $\left(\mathrm{CDCl}_{3}\right): \delta 1.13(3 \mathrm{H}, \mathrm{t}, \mathrm{J}=7.0 \mathrm{~Hz}$, C19-methyl protons), 2.30-2.60 (2H, m, C18-methylene protons), 5.40 (2H, s, C5-methylene protons), 5.46-5.85 (2H, $\mathrm{dd}, \mathrm{J}=17.50,17.55 \mathrm{~Hz}, \mathrm{C} 17-$ methylene protons), $7.25(1 \mathrm{H}, \mathrm{s}$, C14-H), 7.72 (1H, t, J=8.02 Hz, C25-H), $7.88(1 \mathrm{H}, \mathrm{t}, \mathrm{J}=8.01 \mathrm{~Hz}$, C11-H), 8.37-8.52 (4H, m, C10-H, C12-H, C24-H, C26-H), 8.95 (1H, s, C28-H), 9.28 (1H, s, C7-H); ${ }^{13} \mathrm{C} \mathrm{NMR}\left(\mathrm{CDCl}_{3}\right)$ :

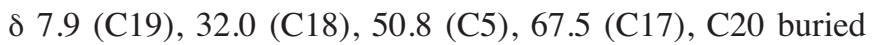
by $\mathrm{CHCl}_{3}$ peaks, 96.7 (C14), 121.0, 121.8, 125.0, 126.1, $127.7,128.5,128.8,130.1,130.8,131.6,135.9,136.5,145.5$, 146.0, 148.2, 148.5, 157.0 (C2, C3, C6-C13, C15, C16, C16a, C23-C28), 163.4, 166.9 (C21,C22); mass m/e (relative intensity): $542\left(\mathrm{~m}^{+}, 3\right), 389$ (20), 375 (100), 360 (38), 347 (78), 332 (58), 306 (30), 286 (261), 272 (15), 258 (10), 229 (8); precise mass $\left(\mathrm{C}_{27} \mathrm{H}_{18} \mathrm{~N}_{4} \mathrm{O}_{9}\right)$ : found 542.107; required, 542.107.

9-Nitrocamptothecin-20-O-p-nitrobenzoate [12]. Using 9-nitrocamptothecin $(0.5 \mathrm{~g}, 0.0013 \mathrm{~mol}), 4$-nitrobenzoic acid (0.5 g, $0.0030 \mathrm{~mol})$, DCC (0.8 g, $0.0039 \mathrm{~mol})$, and DMAP $(0.2 \mathrm{~g}, 0.0016 \mathrm{~mol})$ as the starting materials, pure product $\mathbf{1 2}$ $(0.18 \mathrm{~g})$ was obtained as a yellow powder, yield $26 \%$, purity 99\% (HPLC). ${ }^{1} \mathrm{H} \mathrm{NMR}\left(\mathrm{CDCl}_{3}\right): \delta 1.11(3 \mathrm{H}, \mathrm{t}, \mathrm{J}=7.05 \mathrm{~Hz}$, C19-methyl protons), 2.20-2.53 (2H, m, C18-methylene protons), 5.39 ( $2 \mathrm{H}, \mathrm{s}, \mathrm{C} 5$-methylene-protons), 5.40-5.83 (2H, $\mathrm{dd}, \mathrm{J}=17.50,17.54 \mathrm{~Hz}, \mathrm{C} 17$-methylene protons), $7.22(1 \mathrm{H}, \mathrm{s}$, $\mathrm{C} 14-\mathrm{H}), 7.86[1 \mathrm{H}, \mathrm{t}(\mathrm{d}+\mathrm{d}), \mathrm{J}=8.03 \mathrm{~Hz}, \mathrm{C} 11-\mathrm{H}], 8.20-8.60$ (6H, m, C10-H, C12-H, C24-H, C25-H, C27-H, and C28-H), $9.25(1 \mathrm{H}, \mathrm{s}, \mathrm{C} 7-\mathrm{H}) ;{ }^{13} \mathrm{C} \mathrm{NMR}\left(\mathrm{CDCl}_{3}\right)$ : $\delta 7.9(\mathrm{C} 19), 32.1$ (C5), 67.3 (C17), C20 buried by solvent peaks in the area of 76.0-78.0 ppm, 96.6 (C14), 121.0, 121.8, 123.9, 126.0, 127.8, $128.8,130.3,131.4,131.5,133.9,136.5,137.0,145.1,146.0$, 148.5, 151.4, 153.5, 157.0 (C2, C3, C6-C13, C15, C16, C16a, C23-C28), 163.6, 166.6 (C21, C22); mass m/e (relative intensity): $542\left(\mathrm{~m}^{+}, 8\right), 375(95), 347(100), 333$ (14), 304 (8), 258 (6), 203 (4); Precise mass $\left(\mathrm{C}_{27} \mathrm{H}_{18} \mathrm{~N}_{4} \mathrm{O}_{9}\right)$ : found, 542.109; required, 542.107.

9-Nitrocamptothecin-20-O-m-cyanobenzoate [13]. Using 9-nitrocamptothecin $(0.5 \mathrm{~g}, 0.0013 \mathrm{~mol}), 3$-cyanobenzoic acid ( $1 \mathrm{~g}, 0.0068 \mathrm{~mol})$, DCC (1.5 g, $0.0073 \mathrm{~mol})$, and DMAP $(0.2 \mathrm{~g}, 0.0016 \mathrm{~mol})$ as the starting materials, pure product $\mathbf{1 3}$ $(0.26 \mathrm{~g})$ was obtained as a yellow powder, yield $38 \%$, purity 99\% (HPLC). ${ }^{1} \mathrm{H}$ NMR $\left(\mathrm{CDCl}_{3}\right): \delta 1.14$ (3H, bs, C19-methyl protons), 2.25-2.26 ( $2 \mathrm{H}, \mathrm{m}, \mathrm{C} 18$-methylene protons), 5.38 ( $2 \mathrm{H}, \mathrm{s}, \mathrm{C5}$-methylene protons), 5.40-5.78 (2H, dd $\mathrm{J}=17.50$, $17.54 \mathrm{~Hz}, \mathrm{C} 17-$-methylene protons), $7.21(1 \mathrm{H}, \mathrm{s}, \mathrm{C} 14-\mathrm{H})$, 7.60-7.70 (1H, m, C25-H), 7.80-7.96 (2H, m, C11-H, C24H), 8.25-8.55 (4H, m, C10-H, C12-H, C26-H, C28-H), 9.28 $(1 \mathrm{H}, \mathrm{s}, \mathrm{C} 7-\mathrm{H}) ;{ }^{13} \mathrm{C} \mathrm{NMR}\left(\mathrm{CDCl}_{3}\right): 7.9(\mathrm{C} 19), 32.5(\mathrm{C} 18)$, 50.8 (C5), $67.5(\mathrm{C} 17), \mathrm{C} 20$ buried by $\mathrm{CHCl}_{3}$ peaks in the area of 76.0-78.0 ppm, 96.8 (C14), 113.2, 117.8, 121.0, $121.8,126.1,127.8,128.8,129.9,130.1,131.6,133.9,134.1$, 136.7, 137.0, 145.4, 146.0, 148.4, 153.1, 156.1, 156.5 (C2, C3, C6-C13, C15, C216, C16a, C23-C28, C27-cyano carbon), 163.1, 166.5 (C21, C22); mass m/e (relative intensity): $522\left(\mathrm{~m}^{+}, 3\right), 389$ (18), 375 (100), 360 (40), 347 (80), 332 (61), 306 (56), 286 (35), 272 (15), 216 (10); precise mass $\left(\mathrm{C}_{28} \mathrm{H}_{18} \mathrm{~N}_{4} \mathrm{O}_{7}\right)$ : found, 522.117; required, 522.118.

9-Nitrocamptothecin-20-O-p-cyanobenzoate [14]. Using 9 -nitrocamptothecin $(0.58 \mathrm{~g}, 0.0015 \mathrm{~mol}), 4$-cyanobenzoic 
acid (1 g, $0.0068 \mathrm{~mol})$, DCC (1.5 g, $0.0073 \mathrm{~mol})$, and DMAP $(0.2 \mathrm{~g}, 0.0016 \mathrm{~mol})$ as the starting materials, pure product 14 $(0.3 \mathrm{~g})$ was obtained as a yellow powder, yield $38 \%$, purity 99\% (HPLC). ${ }^{1} \mathrm{H}$ NMR $\left(\mathrm{CDCl}_{3}\right): \delta 1.15(3 \mathrm{H}, \mathrm{t}, \mathrm{J}=7.05 \mathrm{~Hz}$, C19-methyl protons), 2.30-2.60 (2H, m, C18-methylene protons), 5.44 ( $2 \mathrm{H}, \mathrm{s}, \mathrm{C} 14-\mathrm{H}), 5.55-5.85(2 \mathrm{H}, \mathrm{dd}, \mathrm{J}=17.50$, $17.53 \mathrm{~Hz}, \mathrm{C} 17$-methylene protons), 7.30 (1H, s, C14-H), 7.85 $(2 \mathrm{H}, \mathrm{d}, \mathrm{J}=8.08 \mathrm{~Hz}, \mathrm{C} 24-\mathrm{H}, \mathrm{C} 28-\mathrm{H}, 7.94(1 \mathrm{H}, \mathrm{t}, \mathrm{J}=8.02 \mathrm{~Hz}$, C11-H), 8.26 (2H, d, J=8.09 Hz, C25-H, C27-H), 8.45-8.55 (2H, m, C10-H, C12-H), 9.33 (1H, s, C7-H); ${ }^{13} \mathrm{C}$ NMR $\left(\mathrm{CDCl}_{3}\right)$ : 88.0 (C19), 32.0 (C18), 50.6 (C5), 67.5 (C17), C20 buried by solvent peaks in the area of 76.0-78.0 ppm, 96.6 (C14), 117.2 117.3, 121.0, 121.4, 126.0, 127.5, 128.5, 130.6, 131.6, 132.7, 136.7, 145.2, 145.9, 149.0, 153.9, 157.4, (C2, C3, C6-C13, C15, C16, C16a, C23-C28, C26-cyano carbon), 163.6, 166.8 (C21, C22); mass m/e (relative intensity): 522 $\left(\mathrm{m}^{+}, 2\right), 389(4), 375(100), 360$ (35), 347 (85), 332 (55), 306 (20), $286(25), 272(8), 229$ (5), 203 (2); precise mass $\left(\mathrm{C}_{28} \mathrm{H}_{18} \mathrm{~N}_{4} \mathrm{O}_{7}\right)$ : found, 522.118; required, 522.118.

9-Nitrocamptothecin-20-O-o-fluorobenzoate [15]. Using 9-nitrocamptothecin (0.5 g, $0.0013 \mathrm{~mol}), 2$-fluorobenzoic acid $(1 \mathrm{~g}, 0.0071 \mathrm{~mol})$, DCC (1.5 g, $0.0073 \mathrm{~mol})$, and DMAP $(0.2 \mathrm{~g}, 0.0016 \mathrm{~mol})$ as starting reaction materials, pure product $15(0.12 \mathrm{~g})$ was obtained as a yellow powder, yield $18 \%$, purity $99 \%$ (HPLC). ${ }^{1} \mathrm{H}$ NMR $\left(\mathrm{CDCl}_{3}\right): \delta 1.12(3 \mathrm{H}, \mathrm{t}$, $\mathrm{J}=7.08 \mathrm{~Hz}, \mathrm{C} 19$-methyl protons), 2.20-2.50 (2H, m, C18methylene protons), 5.39 ( $2 \mathrm{H}, \mathrm{s}, \mathrm{C} 5$-methylene protons), 5.45-5.84 (2H, dd, J=17.51, 17.58 Hz, C17-methylene protons), 7.24 (1H, s, C14-H), 7.16-7.40 (2H, m, C24-H, C27-H), 7.52-7.70 (1H, m, C25-H), $7.89(1 \mathrm{H}, \mathrm{t}, \mathrm{J}=8.09 \mathrm{~Hz}$, C11-H), 8.05 (1H, t, J=6.8HZ, C26-H), 8.36-8.60 (2H, m, C10-H, C12-H), 9.28 (1H, s, C7-H); ${ }^{13} \mathrm{C} \mathrm{NMR}\left(\mathrm{CDCl}_{3}\right): \delta 7.9$ (C19), 32.0 (C18), 50.6 (C5), 67.4 (C17), C20 buried by $\mathrm{CHCl}_{3}$ peaks, $97.1(\mathrm{Cl} 4), 117.0,117.6,121.0,121.6,124.4$, $125.9,127.4,128.7,131.4,122.8,135.8,136.7,145.0,146.0$, 148.6, 157.2, 160.8 (C2, C3, C6-C13, C15, C16, C16a, C23$\mathrm{C} 28), 164.5,167.2$ (C22, C22); mass $\mathrm{m} / \mathrm{e}$ (relative intensity): $515\left(\mathrm{~m}^{+}, 2\right), 375$ (22), 347 (18), 332 (8), 286(3), 140 (63), 123 (100), 45 (36), 75 (16); precise mass $\left(\mathrm{C}_{27} \mathrm{H}_{18} \mathrm{~N}_{3} \mathrm{O}_{7} \mathrm{~F}\right)$, found, 515.113; required, 515.113.

9-Nitrocamptothecin-20-O-m-fluorobenzoate [16]. Using 9-nitrocamptothecin (0.5 g, $0.0013 \mathrm{~mol}), 3$-fluorobenzoic acid $(1 \mathrm{~g}, 0.0071 \mathrm{~mol})$, DCC (1.5 g, $0.0073 \mathrm{~mol})$, and DMAP $(0.2 \mathrm{~g}, 0.0016 \mathrm{~mol})$ as the starting materials, pure product $\mathbf{1 6}$ $(0.6 \mathrm{~g})$ was obtained as a yellow powder, yield $90 \%$, purity 99\%. ${ }^{1} \mathrm{H}$ NMR $\left(\mathrm{CDCl}_{3}\right): \delta 1.12(3 \mathrm{H}, \mathrm{t}, \mathrm{J}=7.08 \mathrm{~Hz}, \mathrm{C} 19-$ methyl protons), 2.23-2.55 (2H, m, C18-methyl protons), $5.33(2 \mathrm{H}, \mathrm{s}, \mathrm{C} 5$-methylene protons $), 5.42-5.82(2 \mathrm{H}$, dd, $\mathrm{J}=17.50,17.54 \mathrm{~Hz}, \mathrm{C} 17$-methylene protons), 5.42-5.82 (2H, $\mathrm{dd}, \mathrm{J}=17.50,17.541 \mathrm{~Hz}, \mathrm{C} 17$-methylene protons $), 7.25(1 \mathrm{H}$, s, C14-H), 7.30-7.52 (2H, m, C24-H, C26-H), 7.75-7.93 (3H, m, C14-H, C25-H, C28-4), 8.40-8.50 (2H, d, J=8.08 Hz, $\mathrm{C} 10-\mathrm{H}, \mathrm{C} 12-\mathrm{H}), 9.25(1 \mathrm{H}, \mathrm{s}, \mathrm{C} 7-\mathrm{H}) ;{ }^{13} \mathrm{C} \mathrm{NMR}\left(\mathrm{CDCl}_{3}\right): \delta 7.9$ (C19), 32.0 (C18), 50.8 (C5), 67.5 (C17), C20 buried by solvent peaks, 97.0 (C14), 118.0, 118.2, 121.0, 121.4, 122.0, 126.2, 127.4, 128.6, 131.0, 131.2, 131.9, 137.0, 145.0, 145.5, 145.7, 148.5, 153.9, 157.5, 161.0 (C2, C3, C6-C13, C15,
C16, C16a, C23-C28), 164.3, 167.0 (C21, C22); mass m/e (relative intensity): $515\left(\mathrm{~m}^{+}, 7\right), 375(38), 347$ (32), 332 (10), 286 (3), 140 (60), 123 (100), 95 (50), 175 (15); precise mass $\left(\mathrm{C}_{27} \mathrm{H}_{18} \mathrm{~N}_{3} \mathrm{O}_{7} \mathrm{~F}\right)$ : found, 515.133, required, 515.133.

9-Nitrocamptothecin-20-O-p-fluorobenzoate [17]. Using 9-nitrocamptothecin $(0.5 \mathrm{~g}, 0.0013 \mathrm{~mol}), 4$-fluorobenzoic acid (1 g, $0.0071 \mathrm{~mol})$, DCC (1.5 g, $0.0073 \mathrm{~mol})$, and DMAP $(0.2 \mathrm{~g}, 0.0016 \mathrm{~mol})$ as the starting materials, pure product $\mathbf{1 7}$ $(0.15 \mathrm{~g})$ was obtained as a yellow powder, yield $22 \%$, purity $99 \%$ (HPLC). ${ }^{1} \mathrm{H} \mathrm{NMR}\left(\mathrm{CDCl}_{3}\right): \delta 1.10(3 \mathrm{H}, \mathrm{t}, \mathrm{J}=7.04 \mathrm{~Hz}$, C19-methyl protons), 2.20-2.50 (2H, m, C18-methylene protons), 5.38 (2H, s, C5-methylene protons), 5.41-5.82 (2H, $\mathrm{dd}, \mathrm{J}=17.42,17.49 \mathrm{~Hz}, \mathrm{C} 17$-methylene protons), $7.18[2 \mathrm{H}, \mathrm{t}$ $(\mathrm{d}+\mathrm{d}), \mathrm{J}=8.05 \mathrm{~Hz}, \mathrm{C} 25-\mathrm{H}, \mathrm{C} 27-\mathrm{H}], 7.27(1 \mathrm{H}, \mathrm{s}, \mathrm{C} 14-\mathrm{H}), 7.85$ $(1 \mathrm{H}, \mathrm{t}, \mathrm{J}=8.04 \mathrm{~Hz}, \mathrm{C} 11-\mathrm{H}), 8.10-8.20\left(2 \mathrm{H}, \mathrm{d}^{+} \mathrm{d}, \mathrm{J}=8.06,8.05 \mathrm{~Hz}\right.$, C24-H, C28-H), 8.45 (2H, d, J=8.08 Hz, C10-H, C12-H), $9.26(1 \mathrm{H}, \mathrm{s}, \mathrm{C} 7-\mathrm{H}) ;{ }^{13} \mathrm{C} \mathrm{NMR}\left(\mathrm{CDCl}_{3}\right)$ : $\delta 7.9(\mathrm{C} 19), 32.2$ (C18), 50.6 (C5), 67.6 (C17), C20 buried by solvent peaks in the area of 76.0-78.0 ppm, $97.3(\mathrm{C} 14), 116.0,116.3,121.0$, 121.6, 125.0, 126.0, 127.5, 128.0, 131.6, 133.2, 136.3, 145.0, 146.0, 148.6, 153.4, 156.8, 157.0 (C2, C3, C6-C13, C15, C16, C16a, C23-C28), 164.2, 168.1 (C21,C22); mass m/e (relative intensity): $515\left(\mathrm{~m}^{+}, 8\right), 375$ (60), 360 (14), 347 (50), 332 (10), 155 (6), 140 (18), 123 (100), 95(30); precise mass $\left(\mathrm{C}_{27} \mathrm{H}_{18} \mathrm{~N}_{3} \mathrm{O}_{7} \mathrm{~F}\right)$ : found, 515.113; required, 515.113.

9-Nitrocamptothecin-20-O-o-chlorobenzoate [18]. Using 9-nitrocamptothecin $(0.5 \mathrm{~g}, 0.0013 \mathrm{~mol}), 2$-chlorobenzoic acid (1 g, $0.0063 \mathrm{~mol})$, DCC (1.5 g, $0.0073 \mathrm{~mol})$, and AMAP $(0.2 \mathrm{~g}, 0.0016 \mathrm{~mol})$ as the starting materials, pure product $\mathbf{1 8}$ $(0.25 \mathrm{~g})$ was obtained as a yellow powder, yield $36 \%$, purity $99 \%$ (HPLC). ${ }^{1} \mathrm{H} \mathrm{NMR}\left(\mathrm{CDCl}_{3}\right): \delta 1.12(3 \mathrm{H}, \mathrm{t}, \mathrm{J}=7.10 \mathrm{~Hz}$, C19-methyl protons), 2.20-2.50 (2H, m, C18-methylene protons), 5.38 (2H, s, C5-methlene protons), 5.42-5.85 (2H, $\mathrm{dd}, \mathrm{J}=17.51,17.56 \mathrm{~Hz}, \mathrm{C} 17$-methylene protons), $7.38(1 \mathrm{H}, \mathrm{s}$, C14-H), 7.35-7.50 (3H, m, C25-H, C26-H, C27-H), 7.87 (1H, t, $\mathrm{J}=8.08 \mathrm{~Hz}, \mathrm{C} 11-\mathrm{H}), 8.05(\mathrm{lH}, \mathrm{d}, \mathrm{J}=8.06 \mathrm{~Hz}, \mathrm{C} 24-\mathrm{H}), 8.44-8.54$ $(2 \mathrm{H}, \mathrm{d}+\mathrm{d}, \mathrm{J}=8.05,8.08 \mathrm{~Hz}, \mathrm{C} 10-\mathrm{H}, \mathrm{C} 12-\mathrm{H}), 9.26(1 \mathrm{H}, \mathrm{s}, \mathrm{C} 7-$ $\mathrm{H}) ;{ }^{13} \mathrm{C}$ NMR: $\delta 7.9$ (C19), 32.3 (C18), 50.8 (C5), 67.7 (C17), $\mathrm{C} 20$ buried by solvent peaks in the area of 76.0-78.0 ppm, 97.3 (C14), 121.0, 121.2, 125.9, 126.9, 127.4, 128.7, 131.3, 131.6, 132.2, 133.7, 134.8, 136.8, 145.0, 146.0, 148.8, 153.7, 156.6, 158.9 (C2, C3, C6-C13, C15, C16, C16a, C23-C28), 164.0, 166.8 (C21, C22); mass m/e (relative intensity): 531 $\left(\mathrm{m}^{+}\right.$, weak), 375 (100), 360 (20), 347 (85), $335(80), 285$ (38), 235 (38), 185 (8), 147 (75), 139 (85), 111 (18), 97 (25), 77 (15); precise mass $\left(\mathrm{C}_{27} \mathrm{H}_{18} \mathrm{~N}_{3} \mathrm{O}_{7} \mathrm{Cl}\right)$ : found, 531.083; required, 531.083

9-Nitrocamptothecin-20-O-m-chlorobenzoate [19]. Using 9-nitrocamptothecin $(0.5 \mathrm{~g}, 0.0013 \mathrm{~mol}), 3$-chlorobenzoic acid $(0.5 \mathrm{~g}, 0.0032 \mathrm{~mol}), \mathrm{DCC}(0.8 \mathrm{~g}, 0.0039 \mathrm{~mol})$, and DMAP $(0.2 \mathrm{~g}, 0.0016 \mathrm{~mol})$ as the starting materials, pure product $19(0.06 \mathrm{~g})$ was obtained as a yellow powder, yield 9\%, purity $99 \%$ (HPLC). ${ }^{1} \mathrm{H}$ NMR $\left(\mathrm{CDCl}_{3}\right): \delta 1.10(3 \mathrm{H}, \mathrm{t}$, $\mathrm{J}=7.04 \mathrm{~Hz}, \mathrm{C} 19$-methyl protons), 2.20-2.54 (2H, m, C18methyl protons), 5.38 ( $2 \mathrm{H}, \mathrm{s}, \mathrm{C} 5$-methylene protons), 5.40$5.83(2 \mathrm{H}, \mathrm{dd}, \mathrm{J}=17.52,17.55 \mathrm{~Hz}, \mathrm{C} 17$-methylene protons), 
$7.23(1 \mathrm{H}, \mathrm{s}, \mathrm{C} 14-\mathrm{H}), 7.44[1 \mathrm{H}, \mathrm{t}(\mathrm{d}+\mathrm{d}), \mathrm{J}=8.06 \mathrm{~Hz}, \mathrm{C} 25-\mathrm{H}]$, $7.59(1 \mathrm{H}, \mathrm{d}, \mathrm{J}=8.08 \mathrm{~Hz}, \mathrm{C} 26-\mathrm{H}), 7.86[1 \mathrm{H}, \mathrm{t}(\mathrm{d}+\mathrm{d}), \mathrm{J}=8.08 \mathrm{~Hz}$, C11-H], 7.98 (1H, d, J=8.04 Hz, C24-H), 8.10 (1H, s, C28-H), $8.45(2 \mathrm{H}, \mathrm{d}, \mathrm{J}=8.06 \mathrm{~Hz}, \mathrm{C} 10-\mathrm{H}, \mathrm{C} 12-\mathrm{H}), 9.25(1 \mathrm{H}, \mathrm{s}$, $\mathrm{C} 7-\mathrm{H}) ;{ }^{13} \mathrm{C} \mathrm{NMR}\left(\mathrm{CDCl}_{3}\right)$ : $\delta 7.9$ (C19), 32.0 (48), 50.4 (C5), $67.0(\mathrm{C} 17), \mathrm{C} 20$ buried by solvent peaks in the area of 76.0-78.0 ppm, 96.9 (C14), 120.8, 121.5, 125.8, 127.4, 128.3 128.6, 129.7, 129.8, 129.9, 131.2, 134.0, 134.9, 136.6, 144.9, 145.5, 145.8, 148.8, 157.2 (C2, C3, C6-C13, C15, C16, Cl6a, C23-C28), 164.2, 167.1 (C21, C22); mass m/e (relative intensity): $531\left(\mathrm{~m}^{+}, 1\right), 375$ (35), 360 (6), 347 (25), 243 (15), 231 (15), 156 (100), 139 (90), 119 (82), 111 (35), 100 (28), 75 (8); precise mass $\left(\mathrm{C}_{27} \mathrm{H}_{18} \mathrm{~N}_{3} \mathrm{O}_{7} \mathrm{Cl}\right)$ : found, 531.084; required, 531.083 .

9-Nitrocamptothecin-20-O-p-chlorobenzoate [20]. Using 9-nitrocamptothecin $(0.5 \mathrm{~g}, 0.0013 \mathrm{~mol})$, 4-chlorobenzoic acid $(0.5 \mathrm{~g}, 0.0032 \mathrm{~mol})$, DCC (1 g, $0.0049 \mathrm{~mol})$, and DMAP $(0.2 \mathrm{~g}, 0.0016 \mathrm{~mol})$ as the starting materials, pure product 20 $(0.05 \mathrm{~g})$ was obtained as a yellow powder, yield $7 \%$, purity 99\% (HPLC). ${ }^{1} \mathrm{H}$ NMR $\left(\mathrm{CDCl}_{3}\right): \delta 1.11(3 \mathrm{H}, \mathrm{t}, \mathrm{J}=7.06 \mathrm{~Hz}$, C19-methyl protons), 2.20-2.50 (2H, m, C18-methylene protons), $5.35(2 \mathrm{H}, \mathrm{s}, \mathrm{C} 5$-methylene protons), 5.40-5.82 (2H, dd, J=17.51, $17.55 \mathrm{~Hz}, \mathrm{C} 17$-methylene protons), $7.23(1 \mathrm{H}, \mathrm{s}$, C14-H), 7.47 (2H, d, J=8.09 Hz, C25-H, C27-H), $7.86(1 \mathrm{H}, \mathrm{t}$, $\mathrm{J}=8.0 \mathrm{~Hz}, \mathrm{C} 11-\mathrm{H}), 8.04$ (2H, d, J=8.07 Hz, C24-H, C28-H), 8.43 (2H, d, J=8.03 Hz, C10-H, C12-H), 9.25 (1H, s, C7-H); ${ }^{13} \mathrm{C} \mathrm{NMR}\left(\mathrm{CDCl}_{3}\right)$ : $\delta 7.9$ (C19), 50.5 (C5), 67.2 (C17), C20 buried by solvent peaks in the area of 76.0-78.0 ppm, 96.8 (C14), 120.9, 121.5, 125.9, 127.4, 128.8, 129.1, 131.5, 136.5, 140.4, 145.0, 145.8, 145.9, 148.7, 153.6, 157.0 (C2, C3, C6C13, C15, C16, C16a, C23-C28), 164.5, 167.0 (C21, C22); mass $\mathrm{m} / \mathrm{e}$ (relative intensity): $531\left(\mathrm{~m}^{+}\right.$, weak), 375 (95), 360 (35), 347 (70), 332 (38), 156 (43), 139 (100), 111 (35), 75 (10). Precise mass $\left(\mathrm{C}_{27} \mathrm{H}_{18} \mathrm{~N}_{3} \mathrm{O}_{7} \mathrm{Cl}\right)$ : found, 531.083; required, 531.083 .

9-Nitrocamptothecin-20-O-o-bromobenzoate [21]. Using 9-nitrocamptothecin $(0.5 \mathrm{~g}, 0.0013 \mathrm{~mol}), 2$-bromobenzoic acid (1 g, $0.0050 \mathrm{~mol})$, DCC (90.75 g, $0.0036 \mathrm{~mol})$, and DMAP $(0.2 \mathrm{~g}, 0.0016 \mathrm{~mol})$ as the starting materials, pure product $21(0.14 \mathrm{~g})$ was obtained as a yellow powder, yield $19 \%$, purity 99\% (HPLC). ${ }^{1} \mathrm{H}$ NMR (DMSO): $\delta 1.00(3 \mathrm{H}, \mathrm{t}$, $\mathrm{J}=7.06 \mathrm{~Hz}, \mathrm{C} 19$-methyl protons), 2.20-2.40 (2H, m, C18methylene protons), 5.35 ( $2 \mathrm{H}, \mathrm{s}, \mathrm{C} 25$-methylene protons), 5.58 (2H, s, C17-methylene protons), $7.26(1 \mathrm{H}, \mathrm{s}, \mathrm{C} 14-\mathrm{H})$, 7.50-8.60 (7H, m, C10-C12-Hs, C25-C28-Hs), 9.15 (1H, s, C7-H); ${ }^{13} \mathrm{C}$ NMR (DMSO): $\delta 7.6$ (C19), 30.6 (C18), 50.8 (C5), 66.1 (C17), 77.3 (C20), 95.5 (C14), 119.6, 119.9, 121.2, 125.1, 127.3, 128.6, 129.0, 131.8, 132.6, 134.0, 134.5, 136.0, 144.5, 144.8, 146.2, 147.5, 156.6 (C2, C3, C6-C13, C15, C16, C16a, C23-C28), 163.6, 166.4 (C21, C22); mass $\mathrm{m} / \mathrm{e}$ (relative intensity): $577(\mathrm{M}+2,5), 575\left(\mathrm{~m}^{+}, 5\right), 375(58)$, 347 (38), 332 (12), 286 (4), 202 (26), 183 (36), 84 (100); precise mass $\left(\mathrm{C}_{27} \mathrm{H}_{18} \mathrm{~N}_{3} \mathrm{O}_{7} \mathrm{Br}\right)$ : found, 575.032; required 575.033 .

9-Nitrcamptothecin-20-O-m-bromobenzoate [22]. Using 9-nitrocamptothecin (0.5 g, $0.0013 \mathrm{~mol}), 3$-bromobenzoic acid (0.5 g, $0.0025 \mathrm{~mol})$, DCC (0.75 g, $0.0036 \mathrm{~mol})$, and DMAP $(0.2 \mathrm{~g}, 0.0016 \mathrm{~mol})$ as the starting materials, pure product $22(0.06 \mathrm{~g})$ was obtained as a yellow powder, yield $8 \%$, purity $99 \%$ (HPLC). ${ }^{1} \mathrm{H}$ NMR $\left(\mathrm{CDCl}_{3}\right): \delta 1.10(3 \mathrm{H}, \mathrm{t}$, $\mathrm{J}=7.04 \mathrm{~Hz}, \mathrm{C} 19$-methyl protons), 2.20-2.52 (2H, m, C18methylene protons), 5.40 ( $2 \mathrm{H}, \mathrm{s}, \mathrm{C} 5$-methylene protons), 5.41-5.85 (2H, dd, J=17.50, 17.55 Hz, C17-methylene protons), $7.24(1 \mathrm{H}, \mathrm{s}, \mathrm{C} 14-\mathrm{H}), 7.36(1 \mathrm{H}, \mathrm{t}, \mathrm{J}=8.03 \mathrm{~Hz}, \mathrm{C} 25-\mathrm{H})$, $7.76(1 \mathrm{H}, \mathrm{d}, \mathrm{J}=8.04 \mathrm{~Hz}, \mathrm{C} 26-\mathrm{H}), 7.88(1 \mathrm{H}, \mathrm{t}, \mathrm{J}=8.05 \mathrm{~Hz}, \mathrm{C} 11-$ H), 8.05 (1H, d, J=8.06 Hz, C24-H), 8.26 (1H, s, C28-H), 8.48 $(2 \mathrm{H}, \mathrm{d}, \mathrm{J}=8.05 \mathrm{~Hz}, \mathrm{C} 10-\mathrm{H}, \mathrm{C} 12-\mathrm{H}), 9.25(1 \mathrm{H}, \mathrm{s}, \mathrm{C} 7-\mathrm{H})$; ${ }^{13} \mathrm{C} \mathrm{NMR}\left(\mathrm{CDCl}_{3}\right): \delta 7.9$ (C19), 50.4 (C5), 67.5 (C17), C20 buried by $\mathrm{CHCl}_{3}, 96.9$ (C14), 121.0, 121.8, 122.5, 125.9, 127.6, 128.7, 128.9, 130.2, 130.5, 131.3, 133.1, 136.6, 137.0, $145.1,145.7,145.9,148.5,153.5,157.0,157.6$ (C2, C3, C6C13, C15, C16, C16a, C23-C28), 164.0, 167.0 (C21, C22); mass $\mathrm{m} / \mathrm{e}$ (relative intensity): $575\left(\mathrm{~m}^{+}, 5\right), 389(10), 375$ (100), 360 (35), 347 (74), 332 (48), 318 (8), 286 (16), 258 (8), 224 (8). Precise mass $\left(\mathrm{C}_{27} \mathrm{H}_{18} \mathrm{~N}_{3} \mathrm{O}_{7} \mathrm{Br}\right)$ : found, 575.032; required, 575.034 .

9-Nitrocamptothecin-20-O-o-hydroxylbenzoate [23]. Using 9-nitrocamptothecin $(0.5 \mathrm{~g}, 0.0013 \mathrm{~mol}), 2$-hydroxylbenzoic acid (0.5 g, $0.0036 \mathrm{~mol})$, DCC (0.75 g, $0.0036 \mathrm{~mol})$, and DMAP $(0.2 \mathrm{~g}, 0.0016 \mathrm{~mol})$ as the starting materials, pure product $23(0.03 \mathrm{~g})$ was obtained as a yellow powder, yield $5 \%$, purity $99 \%$ (HPLC). ${ }^{1} \mathrm{H}$ NMR $\left(\mathrm{CDCl}_{3}\right): \delta 1.11(3 \mathrm{H}, \mathrm{t}$, $\mathrm{J}=7.05 \mathrm{~Hz}, \mathrm{C} 19$-methyl protons), 2.20-2.52 (2H, m, C18methylene protons), 5.40 ( $2 \mathrm{H}, \mathrm{s}, \mathrm{C} 5$-methylene protons), 5.41-5.85 (2H, dd, J=17.51, 17.55 Hz, C17-methylene protons), 6.80-7.06 $(2 \mathrm{H}, \mathrm{m}, \mathrm{C} 25-\mathrm{H}, \mathrm{C} 27-\mathrm{H}), 7.24(1 \mathrm{H}, \mathrm{s}$, C14-H), $7.55[1 \mathrm{H}, \mathrm{t}(\mathrm{d}+\mathrm{d}), \mathrm{J}=8.02 \mathrm{~Hz}, \mathrm{C} 26-\mathrm{H}], 7.90[1 \mathrm{H}, \mathrm{t}$ $(\mathrm{d}+\mathrm{d}), \mathrm{J}=8.05 \mathrm{~Hz}, \mathrm{C} 11-\mathrm{H}], 8.10(1 \mathrm{H}, \mathrm{d}, \mathrm{J}=8.03 \mathrm{~Hz}, \mathrm{C} 24-\mathrm{H})$, 8.45 (2H, d, J=8.04 Hz, Cl0-H, C12-H), 9.25 (1H, s, C7-H), $10.0\left(1 \mathrm{H}, \mathrm{s}, \mathrm{C} 28\right.$-phinolic proton); ${ }^{13} \mathrm{C} \mathrm{NMR}\left(\mathrm{CDCl}_{3}\right): \delta 7.9$ (C19), 32.0 (C18), 50.3 (C5), 67.5 (C17), C20 buried by solvent peaks in the area of 76.0-78.0 ppm, 96.9 (C14), $111.0,118.1,119.7,121.0,121.6,126.0,127.5,128.8,130.2$, 131.3, 137.0, 137.2, 145.0, 145.2, 145.5, 148.5, 153.5, 157.3 (C2, C3, C6-C13, C15, C16, C16a, C23-C28), 162.5, 168.6 (C21, C22); mass m/e (relative intensity): $513\left(\mathrm{~m}^{+}, 1\right), 375$ (25), 347 (12), 138 (62), 120 (100), 92 (56), 64 (10). Precise mass $\left(\mathrm{C}_{27} \mathrm{H}_{19} \mathrm{~N}_{3} \mathrm{O} 8\right)$ : found, 513.116; required, 513.117.

9-Nitrocamptothecin-20-O-o,p-dinitrobenzoate [24]. Using 9-nitrocamptothecin $(0.8 \mathrm{~g}, 0.0020 \mathrm{~mol}), 2,4$-dinitrobenzoic acid (2 g, $0.0094 \mathrm{~mol})$, DCC (1 g, $0.0049 \mathrm{~mol})$, and DMAP $(0.2 \mathrm{~g}, 0.0016 \mathrm{~mol})$ as the starting materials, pure product $\mathbf{2 4}$ $(0.1 \mathrm{~g})$ was obtained as a yellow powder, yield $9 \%$, purity $99 \%$ (HPLC). ${ }^{1} \mathrm{H} \mathrm{NMR}\left(\mathrm{CDCl}_{3}\right): \delta 1.08(3 \mathrm{H}, \mathrm{t}, \mathrm{J}=7.56 \mathrm{~Hz}$, C19-methyl protons), 2.15-2.40 (2H, m, C-18-methylene protons), 5.40 (2H, s, C5-methylene protons), 5.41-5.85 (2H, $\mathrm{dd}, \mathrm{J}=17.50,17.58 \mathrm{~Hz}, \mathrm{C} 17$-methylene protons), $7.58(1 \mathrm{H}, \mathrm{s}$, C14-H), 7.94 (1H, t, J=8.08 Hz, C11-H), $8.15(1 \mathrm{H}, \mathrm{d}, \mathrm{J}=8.06 \mathrm{~Hz}$, C10-H), 8.45-8.70 (3H, m, C12-H, C27-H, C28-H), 8.86 $(1 \mathrm{H}, \mathrm{s}, \mathrm{C} 25-\mathrm{H}), 9.28(1 \mathrm{H}, \mathrm{s}, \mathrm{C} 7-\mathrm{H}) ;{ }^{13} \mathrm{C} \mathrm{NMR}\left(\mathrm{CDCl}_{3}\right): \delta 7.9$ (C19), 31.6 (C18), 51.0 (C5), 67.5 (C17), 79.0 (C20), 97.5 (C14), 119.8, 121.4, 126.0, 127.4, 128.0, 128.6, 131.6, 131.8, 132.2 , 137.0, 145.5, 145.8, 146.0, 147.5, 149.2, 149.4, 153.5, 
157.4 (C2, C3, C6-C13, C15, C16, C16a, C23-C28), 164.0, 167.8 (C21, C22); mass m/e (relative intensity): $587\left(\mathrm{~m}^{+}\right.$, weak), 389 (4), 377 (6), 347 (6), 306 (5), 212 (75), 168 (100), $120(85), 75(70)$. Precise mass $\left(\mathrm{C}_{27} \mathrm{H}_{18} \mathrm{~N}_{5} \mathrm{O}_{11}\right)$ : found, 587.092; required, 587.092 .

9-Nitrocamptothecin-20-O-m,m-dinitrobenzoate [25]. Using 9-nitrocamptothecin (0.8 g, $0.0020 \mathrm{~mol}), 3,5$-dinitrobenzoic acid $(1.5 \mathrm{~g}, 0.0071 \mathrm{~mol})$, DCC (1.3 g, $0.0063 \mathrm{~mol})$, and DMAP $(0.3 \mathrm{~g}, 0.0025 \mathrm{~mol})$ as the starting materials, pure product $25(0.9 \mathrm{~g})$ was obtained as a yellow powder, yield $77 \%$, purity $99 \%$ (HPLC). ${ }^{1} \mathrm{H}$ NMR $\left(\mathrm{CDCl}_{3}\right): \delta 1.10(3 \mathrm{H}, \mathrm{t}$, $\mathrm{J}=7.50 \mathrm{~Hz}, \mathrm{C} 19$-methyl protons), 2.30-2.60 (2H, m, C18methylene protons), 5.37 ( $2 \mathrm{H}, \mathrm{s}, \mathrm{C} 2$-methylene protons), 5.40-5.84 (2H, dd, J=17.50, 17.55 Hz, C17-methylene protons), 7.20 (1H, s, C14-H), $7.88(1 \mathrm{H}, \mathrm{t}, \mathrm{J}=8.15 \mathrm{~Hz}, \mathrm{C} 11-\mathrm{H})$, 8.35-8.50 [2H, t (dd), J=8.09 Hz, C10-H, C12-H], $9.18(2 \mathrm{H}$, strong s, C24-H, C28-H), 9.30 (2H, s, C7-H, C26-H); ${ }^{13} \mathrm{C}$ NMR $\left(\mathrm{CDCl}_{3}\right): \delta 17.8(\mathrm{C} 19), 32.0(\mathrm{C} 18), 50.6(\mathrm{C} 5), 67.6(\mathrm{C} 17)$, 78.8 (C20), 96.5 (C14), 121.0, 122.1, 123.5, 126.4, 128.0, 129.1, 130.0, 131.6, 132.3, 136.4, 144.5, 145.1, 145.8, 149.0, 153.2, 157.4 (C2, C3, C6-C13, C15-C16, C16a, C23-C28), 161.5, 166.4 (C21, C22), mass m/e (relative intensity): 587 (m+ weak), 389 (1), 377 (3), 306 (2), 212 (100), 166 (26), 120 (20), 75 (40). Precise mass $\left(\mathrm{C}_{27} \mathrm{H}_{17} \mathrm{~N}_{5} \mathrm{O}_{11}\right)$ : found, 587.092; required, 587.092 .

9-Nitrocamptothecin-20-O-p-methyl-m,m-dinitrobenzoate [26]. Using 9-nitrocamptothecin $(0.8 \mathrm{~g}, 0.0020 \mathrm{~mol}), 4-$ methyl-3,5-dinitrobenzoic acid (2 g, $0.0088 \mathrm{~mol})$, DCC (1.3 g, $0.0063 \mathrm{~mol})$, and DMAP $(0.3 \mathrm{~g}, 0.0025 \mathrm{~mol})$ as the starting materials, pure product $26(0.15 \mathrm{~g})$ was obtained as a yellow powder, yield $12 \%$, purity $99 \%$ (HPLC). ${ }^{1} \mathrm{H} \mathrm{NMR}\left(\mathrm{CDCl}_{3}\right)$ :

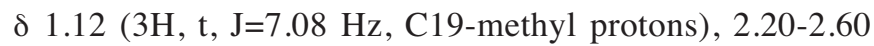
(2H, m, C18-methylene protons), 2.68 (3H, s, C26-methyl protons), 5.40 (2H, s, C5-methylene protons), 5.41-5.84 (2H, $\mathrm{dd}, \mathrm{J}=17.52,17.56 \mathrm{~Hz}, \mathrm{C} 17$-methylene protons $), 7.20(1 \mathrm{H}, \mathrm{s}$, C14-H), 7.90 (1H, t, J=8.08 Hz, C11-H), 8.45 (2H, d, $\mathrm{J}=8.06 \mathrm{~Hz}, \mathrm{C} 10-\mathrm{H}, \mathrm{C} 12-\mathrm{H}), 8.64(2 \mathrm{H}, \mathrm{s}, \mathrm{C} 24-\mathrm{H}, \mathrm{C} 28-\mathrm{H})$, $9.27(1 \mathrm{H}, \mathrm{s}, \mathrm{C} 7-\mathrm{H}) ;{ }^{13} \mathrm{C} \mathrm{NMR}\left(\mathrm{CDCl}_{3}\right): \delta 7.9$ (C19), 15.0 (C26-methyl carbon), 32.4 (C18), 50.5 (C5), 67.4 (C17), 78.0 (C20), 96.8 (C14), 121.0, 121.8, 125.4, 126.0, 127.6, 128.2, $128.3,129.0,129.2,131.5,132.8,136.6,144.5,145.9,146.1$, $148.9,152.1,153.6,157.2$ (C2, C3, C6-C13, C15, C16, C16a, C23-C28, 161.6, 166.5 (C21, C22); mass m/e (relative intensity): 602 (M+1, 45), 449 (100), 376 (40), 347 (12), 332 (15), 136 (15), 72 (46). Precise mass $\left(\mathrm{C}_{28} \mathrm{H}_{20} \mathrm{~N}_{5} \mathrm{O}_{11}\right)$ : found, 602.116; required, 602.116 .

9-Nitrocamptothecin-20-O-m-trifluoromethylbenzoate [27]. Using 9-nitrocamptothecin $(0.5 \mathrm{~g}, 0.0013 \mathrm{~mol}), 3$-trifluoromethylbenzoic acid $(1 \mathrm{~g}, 0.0053 \mathrm{~mol}), \mathrm{DCC}(0.8 \mathrm{~g}$, $0.0039 \mathrm{~mol})$, and DMAP $(0.2 \mathrm{~g}, 0.0016 \mathrm{~mol})$ as the starting materials, pure product $27(0.45 \mathrm{~g})$ was obtained as a yellow powder, yield $61 \%$, purity $99 \%$ (HPLC). ${ }^{1} \mathrm{H} \mathrm{NMR}\left(\mathrm{CDCl}_{3}\right)$ : o $1.10(3 \mathrm{H}, \mathrm{t}, \mathrm{J}=7.08 \mathrm{~Hz}, \mathrm{C} 19$-methyl protons), 2.20-2.50 (2H, m, C18-methylene protons), 5.38 (2H, s, C5-methylene protons), 5.40-5.85 (2H, dd, J=17.51, $17.58 \mathrm{~Hz}, \mathrm{C} 17$-methyl protons), 7.24 (1H, s, C14-H), $7.65(1 \mathrm{H}, \mathrm{t}, \mathrm{J}=8.08 \mathrm{~Hz}, \mathrm{C} 25-\mathrm{H})$,
7.82-7.95 (2H, m, C11-H, C26-H), $8.28(1 \mathrm{H}, \mathrm{d}, \mathrm{J}=8.07 \mathrm{~Hz}$, C24-H), 8.38 (1H, s, C28-H), 8.47 (2H, d, J=8.06 Hz, C10$\mathrm{H}, \mathrm{C} 12-\mathrm{H}), 9.25(1 \mathrm{H}, \mathrm{s}, \mathrm{C} 7-\mathrm{H}) ;{ }^{13} \mathrm{C} \mathrm{NMR}\left(\mathrm{CDCl}_{3}\right): \delta 7.9$ (C19), 32.0 (C18), 50.5 (C5), 67.6 (C17), 76.2 (C27trifluoromethyl carbon), 77.2 (C20), 96.9 (C14), 121.0, 121.4, 126.0, 127.2, 127.5, 128.7, 129.4, 129.8, 130.7, 131.4, 133.3, 136.4, 145.2, 145.3, 148.8, 153.5, 157.0 (C2, C3, C6C13, C15, C16, C16a, C23-C28), 164.0, 167.1 (C21, C22); mass m/e (relative intensity): $565\left(\mathrm{~m}^{+}, 3\right), 375(18), 360(5)$, 34 (18), 332 (4), 190 (68), 173 (100), 145 (85), 95 (8). Precise mass $\left(\mathrm{C}_{28} \mathrm{H}_{18} \mathrm{~N}_{3} \mathrm{O}_{7} \mathrm{~F}_{3}\right)$ : found, 565.110; required, 565.110 .

9-Nitrocamptothecin-20-O-p-trifluoromethylbenzoate [28]. Using 9-nitrocamptothecin ( $0.5 \mathrm{~g}, 0.0013 \mathrm{~mol})$, 4-trifluoromethylbenzoic acid (1 g, $0.0053 \mathrm{~mol})$, DCC (0.8 g, $0.0039 \mathrm{~mol})$, and DMAP (0.2 g, $0.0016 \mathrm{~mol})$ as the starting materials, pure product $28(0.35 \mathrm{~g})$ was obtained as a yellow powder, yield $48 \%$, purity $99 \%$ (HPLC). ${ }^{1} \mathrm{H} \mathrm{NMR}\left(\mathrm{CDCl}_{3}\right): \delta 1.10(3 \mathrm{H}, \mathrm{t}$, $\mathrm{J}=7.05 \mathrm{~Hz}, \mathrm{C} 19$-methyl protons), 2.20-2.52 (2H, m, C18methylene protons), 5.39 ( $2 \mathrm{H}, \mathrm{s}, \mathrm{C} 5$-methylene protons), 5.40-5.82 (2H, dd, J=17.50, $17.55 \mathrm{~Hz}, \mathrm{C} 17$-methylene protons), 7.22 (1H, s, C14-H), $7.78(2 \mathrm{H}, \mathrm{d}, \mathrm{J}=8.03 \mathrm{~Hz}, \mathrm{C} 25-$ $\mathrm{H}, \mathrm{C} 27-\mathrm{H}), 7.86(1 \mathrm{H}, \mathrm{t}, \mathrm{J}=8.04 \mathrm{~Hz}, \mathrm{C} 11-\mathrm{H}), 8.24(2 \mathrm{H}, \mathrm{d}$, $\mathrm{J}=8.06 \mathrm{~Hz}, \mathrm{C} 24-\mathrm{H}, \mathrm{C} 28-\mathrm{H}), 8.45$ (2H, d, J=8.06 Hz, C10-H, $\mathrm{C} 12-\mathrm{H}), 9.25$ (1H, s, C7-H); ${ }^{13} \mathrm{C}$ NMR $\left(\mathrm{CDCl}_{3}\right)$ : $\delta 7.9$ (C19), 32.0 (C18), 50.4 (C5), 67.5 (C17), C20 buried by $\mathrm{CHCl}_{3}$ peaks, 96.8 (C14), 121.0, 121.6, 125.8, 126.0, 127.5, 128.7, 130.6, 131.5, 131.9, 136.5, 145.0, 145.2, 145.8, 148.5, 153.5, 157.0 (C2, C3, C6-C13, C15, C16, C16a, C23-C28), 164.7, 166.8 (C21, C22); mass m/e (relative intensity): $565\left(\mathrm{~m}^{+}, 4\right)$, 375 (50), 360 (20), 347 (48), 332 (15), 302 (6), 190 (45), 173 (100), 145 (60), 95 (4). Precise mass $\left(\mathrm{C}_{28} \mathrm{H}_{18} \mathrm{~N}_{3} \mathrm{O}_{7} \mathrm{~F}_{3}\right)$ : found, 565.109; required, 565.110 .

Determination of drug-induced antiproliferative activity and toxicity in cultured cells. To assess the antiproliferative activity of the various ester drugs, identical cell cultures were treated with equimolar concentrations of these esters and the cell number per $\mathrm{ml}$ was counted. Stocks consisted of a fine suspension of esters in polyethylene glycol (PEG-400; Aldrich). Control cultures received only the carrier. The cell number was counted at 24, 72 and $120 \mathrm{~h}$ of treatment. Control compounds included the parental compounds 1a and 1b for positive control. The targeted cells included the following 14 human cancer cell lines: breast (CLO and KIE), colon (McCN and HT-29), lung (SPA and DOY), melanoma (BRO and SB1B), ovarian (EFO-27 and 2774), pancreatic (MIA and Panc-1) and prostate (PC-3 and DU-145). The average response of ester compounds to 14 human cancer cell lines is shown in Tables I and II.

\section{HPLC procedure for purity analysis of ester products 2-29}

Instrumentation. The HPLC system consisted of a Beckman 421 controller with two 110A pumps and a 2-ml injection loop. The UV detector was a SPD-110AV model (Shimadzu, Kyoto, Japan). The HPLC detector was set to monitor the UV absorbance at $220 \mathrm{~nm}$. The integrating software used for the analyses was EZChrome (Shimadzu, 
Table I. Average response of 14 malignant cell lines to $200 \mathrm{nM}$ of esters $\mathbf{2 - 2 8}$.

\begin{tabular}{|c|c|c|c|c|c|c|}
\hline \multirow{2}{*}{ Compounds } & \multicolumn{6}{|c|}{ Time (Day) } \\
\hline & 3 & & 5 & & 7 & \\
\hline Control & 4.00 & & 4.00 & & 4.00 & \\
\hline 2 & 4.00 & $(0 \%)$ & 4.00 & $(0 \%)$ & 4.00 & $(0 \%)$ \\
\hline 3 & 3.93 & $(2 \%)$ & 3.93 & $(2 \%)$ & 3.86 & $(3 \%)$ \\
\hline 4 & 3.86 & $(3 \%)$ & 3.64 & $(9 \%)$ & 3.07 & $(23 \%)$ \\
\hline 5 & 3.71 & $(7 \%)$ & 3.07 & $(23 \%)$ & 2.43 & $(39 \%)$ \\
\hline 6 & 1.93 & $(52 \%)$ & 1.21 & $(70 \%)$ & 0.93 & $(77 \%)$ \\
\hline 7 & 3.57 & $(11 \%)$ & 2.93 & $(27 \%)$ & 2.29 & $(23 \%)$ \\
\hline 8 & 2.79 & $(30 \%)$ & 2.07 & $(48 \%)$ & 1.36 & $(66 \%)$ \\
\hline 9 & 4.00 & $(0 \%)$ & 4.00 & $(0 \%)$ & 4.00 & $(0 \%)$ \\
\hline 10 & 4.00 & $(0 \%)$ & 3.93 & $(2 \%)$ & 3.79 & $(5 \%)$ \\
\hline 11 & 2.36 & $(41 \%)$ & 1.50 & $(63 \%)$ & 1.00 & $(75 \%)$ \\
\hline 12 & 2.71 & $(32 \%)$ & 2.00 & $(50 \%)$ & 1.00 & $(75 \%)$ \\
\hline 13 & 3.20 & $(20 \%)$ & 2.60 & $(35 \%)$ & 1.90 & $(53 \%)$ \\
\hline 14 & 2.80 & $(30 \%)$ & 2.00 & $(50 \%)$ & 1.30 & $(68 \%)$ \\
\hline 15 & 3.60 & $(10 \%)$ & 3.30 & $(18 \%)$ & 3.10 & $(23 \%)$ \\
\hline 16 & 3.90 & $(2 \%)$ & 3.90 & $(2 \%)$ & 3.90 & $(2 \%)$ \\
\hline 17 & 4.00 & $(0 \%)$ & 3.90 & $(2 \%)$ & 3.90 & $(2 \%)$ \\
\hline 18 & 4.00 & $(0 \%)$ & 4.00 & $(0 \%)$ & 4.00 & $(0 \%)$ \\
\hline 19 & 4.00 & $(0 \%)$ & 3.86 & $(3 \%)$ & 4.00 & $(0 \%)$ \\
\hline 20 & 4.00 & $(0 \%)$ & 3.93 & $(2 \%)$ & 3.93 & $(2 \%)$ \\
\hline 21 & 4.00 & $(0 \%)$ & 4.00 & $(0 \%)$ & 4.00 & $(0 \%)$ \\
\hline 22 & 4.00 & $(0 \%)$ & 3.97 & $(1 \%)$ & 3.50 & $(13 \%)$ \\
\hline 23 & 3.14 & $(21 \%)$ & 1.93 & $(52 \%)$ & 1.43 & $(64 \%)$ \\
\hline 24 & 2.64 & $(34 \%)$ & 1.86 & $(54 \%)$ & 1.43 & $(64 \%)$ \\
\hline 25 & 1.86 & $(53 \%)$ & 1.21 & $(70 \%)$ & 0.86 & $(79 \%)$ \\
\hline 26 & 1.71 & $(57 \%)$ & 1.29 & $68 \%)$ & 0.93 & $(77 \%)$ \\
\hline 27 & 3.86 & $(3 \%)$ & 3.14 & $(21 \%)$ & 2.14 & $(46 \%)$ \\
\hline 28 & 3.64 & $(9 \%)$ & 2.79 & $(30 \%)$ & 2.07 & $(48 \%)$ \\
\hline
\end{tabular}

Japan) and Flo-Onelbeta (Radiomatic Instruments, Meridian, CT). A C-8 Microsorb was from Rainin Instruments (Woburn, MA).

HPLC analysis. Reverse-phase HPLC analysis of the samples was performed using an acetonitrile-acetic acidwater mobile phase system. Analyses were carried out at room temperature with a flow rate of $1 \mathrm{ml} / \mathrm{min}$. The solution with a concentration of approximately $0.1 \mathrm{mg} / \mathrm{ml}$ of sample in acetonitrile was prepared by dissolving it in the solvent. A $300-\mu 1$ portion of this solution was taken and added to a $700-\mu 1$ solution of $0.1 \%$ acetic acid in water. After shaking for $\sim 10 \mathrm{sec}, 100 \mu 1$ of this solution was injected through a 2-ml loop onto a column and chromatographed with $70 \%$ water with $0.1 \%$ acetic acid and $30 \%$ acetonitrile as the mobile phase for the first $5 \mathrm{~min}$, and then the gradient of the mobile phase was programmatically increased to $100 \%$ acetonitrile over a period of $4 \mathrm{~min}$. A complete HPLC spectrum was obtained at $15 \mathrm{~min}$. The purity of the sample was determined by measuring the UV peak areas at $254 \mathrm{~nm}$
Table II. Average response of 14 malignant cell lines to $800 \mathrm{nM}$ of esters $\mathbf{2 - 2 8}$.

\begin{tabular}{|c|c|c|c|c|c|}
\hline \multirow{2}{*}{ Compounds } & \multicolumn{5}{|c|}{ Time (Day) } \\
\hline & 3 & & 5 & 7 & \\
\hline Control & 4.00 & & 4.00 & 4.00 & \\
\hline 2 & 3.71 & $(7 \%)$ & $3.86(3 \%)$ & 3.93 & $(2 \%)$ \\
\hline 3 & 3.86 & $(3 \%)$ & $3.14(21 \%)$ & 2.36 & $(41 \%)$ \\
\hline 4 & 3.14 & $(21 \%)$ & $2.14(46 \%)$ & 1.57 & $(61 \%)$ \\
\hline 5 & 2.50 & $(37 \%)$ & $1.86(53 \%)$ & 1.21 & $(70 \%)$ \\
\hline 6 & 1.14 & $(71 \%)$ & $0.79(80 \%)$ & 0.50 & $(88 \%)$ \\
\hline 7 & 2.93 & $(27 \%)$ & $1.79(55 \%)$ & 1.21 & $(70 \%)$ \\
\hline 8 & 2.07 & $(48 \%)$ & $1.21(70 \%)$ & 0.71 & $(82 \%)$ \\
\hline 9 & 4.00 & $(0 \%)$ & $4.00(0 \%)$ & 4.00 & $(0 \%)$ \\
\hline 10 & 3.21 & $(17 \%)$ & $2.50(37 \%)$ & 1.93 & $(52 \%)$ \\
\hline 11 & 1.86 & $(53 \%)$ & $0.86(78 \%)$ & 0.43 & $(89 \%)$ \\
\hline 12 & 1.79 & $(55 \%)$ & $0.79(80 \%)$ & 0.50 & $(88 \%)$ \\
\hline 13 & 2.30 & $(42 \%)$ & $1.40(65 \%)$ & 0.90 & $(78 \%)$ \\
\hline 14 & 2.00 & $(50 \%)$ & $1.10(72 \%)$ & 0.50 & $(88 \%)$ \\
\hline 15 & 2.60 & $(35 \%)$ & $1.60(60 \%)$ & 1.11 & $(72 \%)$ \\
\hline 16 & 3.70 & $(7 \%)$ & $2.90(27 \%)$ & 2.40 & $(40 \%)$ \\
\hline 17 & 3.90 & $(2 \%)$ & $3.90(2 \%)$ & 3.90 & $(2 \%)$ \\
\hline 18 & 4.00 & $(0 \%)$ & $3.93(2 \%)$ & 3.86 & $(3 \%)$ \\
\hline 19 & 3.71 & $(7 \%)$ & $2.64(34 \%)$ & 1.93 & $(52 \%)$ \\
\hline 20 & 3.79 & $(5 \%)$ & $3.57(11 \%)$ & 3.14 & $(22 \%)$ \\
\hline 21 & 4.00 & $(0 \%)$ & $4.00(0 \%)$ & 3.93 & $(2 \%)$ \\
\hline 22 & 3.36 & $(16 \%)$ & $2.50(37 \%)$ & 1.57 & $(61 \%)$ \\
\hline 23 & 2.00 & $(50 \%)$ & $1.00(75 \%)$ & 0.79 & $(80 \%)$ \\
\hline 24 & 1.79 & $(55 \%)$ & $1.29(68 \%)$ & 1.00 & $(75 \%)$ \\
\hline 25 & 1.21 & $(70 \%)$ & $0.71(82 \%)$ & 0.64 & $(84 \%)$ \\
\hline 26 & 1.36 & $(66 \%)$ & $0.71(82 \%)$ & 0.64 & $(84 \%)$ \\
\hline 27 & 2.57 & $(36 \%)$ & $1.64(59 \%)$ & 1.21 & $(70 \%)$ \\
\hline 28 & 2.64 & $(34 \%)$ & $1.86(53 \%)$ & 1.21 & $(70 \%)$ \\
\hline
\end{tabular}

and calculating the percentage associated with the sample peak.

\section{Results and discussion}

The in vitro anticancer activity of esters $\mathbf{2 - 2 8}$ was evaluated with the following 14 different human cancer cell lines: breast (CLO and KIE), colon (McCN and HT-29), lung (SPA and DOY), melanoma (BRO and SB1B), ovarian (EFO-27 and 2774), pancreatic (MIA and Panc-1), and prostate (PC-3 and DU-145). Two concentrations were tested. The data are expressed in growth levels relative to the untreated (control) group. The growth level of the untreated group is defined as level 4, the full growth. Levels 3, 2 and 1 indicate the 75\%, $50 \%$, and $25 \%$ growths relative to the corresponding control groups, respectively. Table I shows the average response of the 14 malignant cell lines to $200 \mathrm{nM}$ of compounds $\mathbf{2 - 2 8}$. Table II shows the average response of the same cell lines to $800 \mathrm{nM}$ of the same compounds. The data in parentheses in 
Table III. Potency of ester products 4-8, 11-15 and 22-28 against 8 different cancer cell lines.

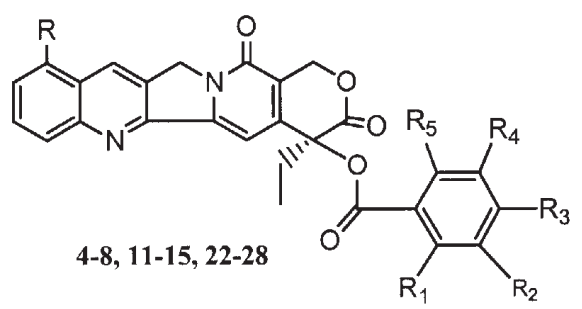

\begin{tabular}{|c|c|c|c|c|c|c|c|c|c|}
\hline \multirow{2}{*}{ Compounds } & \multicolumn{9}{|c|}{ IC50s (nM) } \\
\hline & DOY & Dul45 & $\mathrm{Ht} 29$ & Kielty & $\mathrm{McCN}$ & MPC2 & Sche11 & $\mathrm{T} 47 \mathrm{D}$ & Ave. \\
\hline $\mathrm{CPT}$ & $65 \pm 10$ & $50 \pm 7$ & $46 \pm 25$ & $63 \pm 14$ & $39 \pm 6$ & $41 \pm 4$ & $47 \pm 3$ & $36 \pm 12$ & $48 \pm 10$ \\
\hline $9 \mathrm{NC}$ & $18 \pm 0$ & $15 \pm 1$ & $23 \pm 8$ & $22 \pm 4$ & $36 \pm 13$ & $23 \pm 5$ & $18 \pm 3$ & $11 \pm 3$ & $19 \pm 5$ \\
\hline Irinotecan & $583 \pm 29$ & $572 \pm 20$ & $1099 \pm 54$ & $622 \pm 239$ & $696 \pm 158$ & $589 \pm 87$ & $833 \pm 13$ & $387 \pm 164$ & $673 \pm 96$ \\
\hline Topotecan & $164 \pm 2$ & $158 \pm 43$ & $234 \pm 72$ & $134 \pm 9$ & $125 \pm 3$ & $119 \pm 37$ & $133 \pm 9$ & $96 \pm 24$ & $145 \pm 24$ \\
\hline 4 & $262 \pm 21$ & $316 \pm 44$ & $256 \pm 68$ & $627 \pm 214$ & $810 \pm 215$ & $344 \pm 20$ & $452 \pm 71$ & $445 \pm 80$ & $439 \pm 92$ \\
\hline 5 & $275 \pm 11$ & $302 \pm 25$ & $235 \pm 60$ & $280 \pm 82$ & $1093 \pm 193$ & $284 \pm 21$ & $369 \pm 80$ & $446 \pm 157$ & $410 \pm 78$ \\
\hline 6 & $13 \pm 1$ & $20 \pm 4$ & $15 \pm 0$ & $16 \pm 0$ & $44 \pm 1$ & $15 \pm 2$ & $17 \pm 1$ & $20 \pm 3$ & $20 \pm 2$ \\
\hline 7 & $464 \pm 88$ & $626 \pm 112$ & $508 \pm 186$ & $597 \pm 200$ & $405 \pm 81$ & $659 \pm 47$ & $715 \pm 96$ & $743 \pm 241$ & $590 \pm 113$ \\
\hline 8 & $113 \pm 39$ & $94 \pm 23$ & $110 \pm 25$ & $216 \pm 86$ & $395 \pm 62$ & $112 \pm 20$ & $157 \pm 32$ & $208 \pm 79 /$ & $176 \pm 46$ \\
\hline 11 & $40 \pm 5$ & $92 \pm 8$ & $91 \pm 6$ & $154 \pm 12$ & 1 & $80 \pm 11$ & $76 \pm 12$ & 1 & $89 \pm 9$ \\
\hline 12 & $67 \pm 6$ & $75 \pm 5$ & $128 \pm 18$ & $152 \pm 18$ & $380 \pm 25$ & $125 \pm 9$ & $102 \pm 23$ & $56 \pm 8$ & $136 \pm 14$ \\
\hline 13 & $87 \pm 5$ & $466 \pm 54$ & $966 \pm 98$ & $258 \pm 34$ & $428 \pm 45$ & $242 \pm 24$ & $674 \pm 65$ & $603 \pm 54$ & $466 \pm 47$ \\
\hline 14 & $25 \pm 3$ & $133 \pm 16$ & $578+76$ & $149 \pm 25$ & $146 \pm 32$ & $58 \pm 7$ & $272 \pm 25$ & $430 \pm 46$ & $224 \pm 7$ \\
\hline 15 & $399 \pm 19$ & $289 \pm 32$ & $816 \pm 65$ & $467 \pm 45$ & $500 \pm 67$ & $1065 \pm 98$ & $467 \pm 54$ & $120 \pm 12$ & $515 \pm 49$ \\
\hline 22 & $100 \pm 9$ & $449 \pm 65$ & $624 \pm 76$ & $1840 \pm 96$ & $556 \pm 76$ & $358 \pm 34$ & $1538 \pm 98$ & $781 \pm 76$ & $781 \pm 66$ \\
\hline 23 & $203 \pm 11$ & $152 \pm 13$ & $312 \pm 45$ & $324 \pm 46$ & $750 \pm 87$ & $386 \pm 54$ & $266 \pm 43$ & $306 \pm 32$ & $337 \pm 41$ \\
\hline 24 & $95 \pm 8$ & 1 & $109 \pm 12$ & $98 \pm 10$ & $572 \pm 96$ & $160 \pm 8$ & $94 \pm 16$ & $36 \pm 6$ & $166 \pm 22$ \\
\hline 25 & $10 \pm 3$ & $16 \pm 7$ & $11 \pm 9$ & $16=8$ & $31 \pm 5$ & $12 \pm 8$ & $17 \pm 10$ & $9 \pm 3$ & $15 \pm 7$ \\
\hline 26 & $16 \pm 5$ & $27 \pm 4$ & 1 & $41 \pm 5$ & $92 \pm 9$ & $19 \pm 6$ & $32 \pm 7$ & 1 & $38 \pm 6$ \\
\hline 27 & $73 \pm 10$ & $60 \pm 7$ & 1 & $214 \pm 23$ & 1 & $92 \pm 8$ & $14 \pm 6$ & 1 & $91 \pm 11$ \\
\hline 28 & $922 \pm 237$ & $254 \pm 40$ & $290 \pm 59$ & $431 \pm 131$ & $466 \pm 44$ & $296 \pm 51$ & $345 \pm 85$ & $291 \pm 95$ & $412 \pm 93$ \\
\hline
\end{tabular}

Tables I and II show the corresponding percentage inhibitions, another expression of the anticancer activity of these compounds. The results showed that, at the concentration

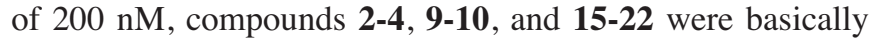
inactive, showing no significant inhibitory effects on the growth of cells. When the concentration was elevated to $800 \mathrm{nM}$, compounds $\mathbf{3}, \mathbf{4}, \mathbf{1 5}, \mathbf{1 6}, \mathbf{1 9}$, and 22 became active. Further analysis of the data in Tables I and II also indicated that the antitumor activity of these esters correlated with the nature of their side aromatic chains. Strong electronwithdrawing groups such as $\mathrm{NO}_{2}, \mathrm{CN}$, and $\mathrm{CF}_{3}$ are beneficial for antitumor activity. For example, esters 6, 8, 11, 12, 13, $\mathbf{1 4}, \mathbf{2 4}, \mathbf{2 5}, \mathbf{2 6}, \mathbf{2 7}$, and 28 were all active against the 14 cell lines tested (Table I). The position where the nitro group attaches did not have any significant effects on antitumor activity. For example, the antitumor activity of esters $\mathbf{1 1}$ and 12 was almost identical, although the nitro groups attached to their side aromatic chains were positioned differently. The antitumor activity of halogen-substituted esters, on the other hand, was clearly dependent on where the halogen atom was attached. For example, as shown in Table II, the $o$-Fsubstituted ester 15 was active, and $m$-F-substituted 16 was slightly active, while $p$-F-substituted $\mathbf{1 7}$ was basically inactive; the $m$-Cl-substituted 19 was slightly active while the $o-\mathrm{Cl}$ - and $p$-Cl-substituted esters $\mathbf{1 8}$ and $\mathbf{2 0}$ were inactive.
Compounds $\mathbf{2}$ and $\mathbf{9}$ were completely inactive against the tested cell lines. The side aromatic chains of these two compounds only contain a plain benzene ring with no substituting groups attached at all. The $\mathrm{OH}$-substituted ester, 23, was also active.

As also shown in Tables I and II, esters 4-8, 11-15, and 22-28 were active against the cancer cells tested. To compare the potency of these active ester compounds with their parental compounds and with commercially available camptothecin analogues, we measured the $\mathrm{IC}_{50}$ values of these esters and that of the control compounds $\mathbf{1 a}, \mathbf{1 b}$, irinotecan, and topotecan in a parallel manner against 8 different human cancer cell lines. The results are shown in Table III. Overall, the potency of the most active ester compounds was lower than that of their parental compounds $\mathbf{1 a}$ and $\mathbf{1 b}$ and higher than the commercial CPT derivatives irinotecan and topotecan. Of all these active compounds, esters $\mathbf{6}$ and $\mathbf{2 5}$ showed remarkable potencies against these 8 cancer cell lines. On average, ester $\mathbf{6}$ was twice as potent as the parental 1a, and 7-33 times more potent than the commercial CPT analogues. Ester 25 was also more potent than the parental $\mathbf{1 b}$ and many times (9-45) more potent than its commercial CPT analogues. Clearly, ester derivatives 6 and $\mathbf{2 5}$ were stronger in activity against these 8 human cancer cell lines than their parental compounds and commercial 
analogues. One explanation for this observation is that these two compounds may themselves be active with no need of the enzymatic cleavage of the ester bond, and if this is the case, these compounds may have different action mechanisms from their parental compounds. Another is that the uptake of these two compounds by the testing cell lines may be easier than that of their parental compounds, and thus the local concentrations of the active metabolites for these two compounds in cells are actually higher than their parental compounds. We think the latter scenario is the more probable. Both of these hypotheses need to be further investigated experimentally, and our institution is undertaking related studies to this end.

In conclusion, the chemistry employed in the preparation of these new aromatic esters is straightforward. The reaction yields vary depending on the nature of the substituting groups on the aromatic side chains. Generally, the reactions with the electron-withdrawing group substituted acids such as nitrobenzoic acids and cyanobenzoic acids as acylating agents, gave good yields. Many ester compounds showed good anticancer activity against the human cancer lines tested. Compounds $\mathbf{6}$ and $\mathbf{2 5}$ are particularly impressive with regard to antitumor activity and potency, and thus have high potential for use as effective agents in cancer treatment. Further biological and preclinical studies such as in vivo antitumor activity and toxicity, pharmacokinetics, and the action mechanism for these two significant camptothecin derivatives are currently being undertaken in our laboratory and the results will be reported.

\section{Acknowledgements}

Supporting funds from the Stehlin Foundation for Cancer Research and the Friends of the Stehlin Foundation are gratefully acknowledged.

\section{References}

1. Wall ME, Wani MC, Cook CE, Palmer K, McPhail AT and Sim GA: Plant antitumor agents. 1. The isolation and structure of camptothecin, a novel alkaloidal leukemia and tumor inhibitor from camptotheca acuminata. J Am Chem Soc 88: 3888-3890, 1966.

2. Gottlieb JA, Guarino AM, Call JB, Oliverio VT and Block JB: Preliminary pharmacologic and clinical evaluation of camptothecin sodium (NSC-100880). Cancer Chemother Rep 54: 461-470, 1970.

3. Gottlieb JA and Luce JK: Treatment of malignant melanoma with camptothecin (NSC-100880). Cancer Chemother Rep 56: 103-105, 1972.

4. Muggia FM, Creaven PJ, Hansen HH, Cohen MH and Selawry OS: Phase I clinical trial of weekly and daily treatment with camptothecin (NSC-100880): Correlation with preclinical studies. Cancer Chemother Rep 56: 515-521, 1972.

5. Moertel CG, Schutt AJ, Reitemeier RJ and Hahn RG: Phase II study of camptothecin (NSC-100880) in the treatment of advanced gastrointestinal cancer. Cancer Chemother Rep 56: 95-101, 1972.

6. Schaeppi U, Fleishman RW and Cooney DA: Toxicity of camptothecin (NSC-100880). Cancer Chemother Rep 58: 25-36, 1974.

7. Wani MC, Ronman PE, Lindley LT and Wall ME: Plant antitumor agents. 18. Synthesis and biological activity of camptothecin analogs. J Med Chem 23: 554-560, 1980.
8. Wall ME and Wani MC: Antineoplastic agents from plants. Annu Rev Pharmacol Toxicol 17: 117-132, 1977.

9. Giovanella BC, Hinz HR, Kozielski AJ, Stehlin JS, Silber R and Potmesil M: Complete growth inhibition of human cancer xenografts in nude mice by treatment with 20-(S)-camptothecin. Cancer Res 51: 3052-3055, 1991.

10. Natelson E, Giovanella BC, Verschraegen CF, Fehir KM, De Ipolyi PD, Harris N and Stehlin JS: Phase I clinical and pharmacological studies of 20-(S)-camptothecin and 20-(S)-9nitrocamptothecin as anticancer agents. Ann NY Acad Sci 803: 224-230, 1996.

11. Burke TG: Chemistry of the camptothecins in the bloodstream: Drug stabilization and optimization of activity. Ann NY Acad Sci 803: 29-31, 1996.

12. Zhao H, Lee C, Sai P, Choe YH, Boro M, Pendri A, Duan S and Greenwald RB: 20-O-acylcamptothecin derivatives: evidence for lactone stabilization. J Org Chem 65: 4601-4606, 2000

13. Burke TG and Mi Z: Preferential binding of the carboxylate form of camptothecin by human serum albumin. Anal Biochem 212: 285-287, 1993

14. Burke TG and Mi Z: The structural basis of camptothecin interactions with human albumin: impact on drug stability. J Med Chem 37: 40-46, 1994.

15. Cao Z, Harris N, Kozielski A, Vardeman D and Giovanella B: Alkyl esters of camptothecin and 9-nitrocamptothecin: Synthesis, in vitro pharmacokinetics, toxicity, and antitumor activity. J Med Chem 41: 31-37, 1998.

16. Cao Z, Pantazis P, Mendoza J, Early J, Kozieslki A, Harris N, Vardeman D, Liehr J, Stehlin J and Giovanella B: Structureactivity relationship of alkyl camptothecin esters. Ann NY Acad Sci 992: 122-135, 2000.

17. Cao Z, Pantazis P, Mendoza J, Early J, Kozielski A, Harris N and Giovanella B: Structure-activity relationship of alkyl 9nitrocamptothecin esters. Acta Pharmacol Sin 24: 109-119, 2003.

18. Kono A and Hara Y: Conversion of CPT-11 into SN-38 in human tissues. Jpn J Cancer Chemother 18: 2175-2178, 1991.

19. Kawato Y, Aonuma M, Matsumoto K and Sato K: Production of SN-38, a main metabolite of the camptothecin derivative CPT-11. Yakubutsu Dotai 6: 899-907, 1991.

20. Satoh T, Hosokawa M, Atsumi R, Suzuki W, Hakusui H and Nagai E: Metabolic activation of CPT-11. Biol Pharm Bull 17: 662-664, 1994.

21. Rivory LP, Bowles MR, Robert J and Pond SM: The conversion of irinotecan (CPT-11) to its active metabolite 7-ethyl-10hydroxycamptothecin (SN38). Biochem Pharmacol 52: 1103-1111, 1996.

22. Mentlein R, Heiland S, and Heymann E: Simultaneous purification and comparative characterization of six serine hydrolases from rat liver microsomes. Arch Biochem Biophys 200: $547-559,1980$

23. Mentlein R and Heymann E: Hydrolysis of ester- and amidetype drugs by the purified isoenzymes of nonspecific carboxylesterase from rat liver. Biochem Pharmacol 33: 1243-1248, 1984.

24. Neises B and Steglich W: Esterification of carboxylic acids with dicyclohexylcarbodiimide/4-dimethylaminopyridine:tert-butyl ethyl fumarate. Org Synth 63: 183-187, 1985.

25. Hassner A and Alexanian V: Direct room temperature esterification of carboxylic acids. Tetrahedron Lett 19: 4475-4478, 1978.

26. Ziegler FE and Berger GD: A mild method for the esterification of fatty acids. Synth Commun 9: 539-543, 1979.

27. Steglich W and Höfle G: N,N-dimethyl-4-pyridinamine, a very effective acylation catalyst. Angew Chem Int Ed Engl 8: 981, 1969.

28. Rao VB and Garzon-Aburbeh A: Water soluble prodrugs of camptothecin. US Patent: 4,943,579, 1990.

29. Wall ME and Wani MC: Water-soluble esters of camptothecin compounds: Non-toxic camptothecin prodrugs are prepared by esterifying the 20-position hydroxyl group of camptothecin derivatives. US Patent: 5,646,159, 1997.

30. Cao Z, Armstrong K, Shaw M, Petry E and Harris N: Nitration of camptothecin with various inorganic nitrate salts in concentrated sulfuric acid: a new preparation of anticancer drug 9-nitrocamptothecin. Synthesis pp1724-1730, 1998. 